\title{
Influence of Electron-Acoustic-Phonon Scattering on Intensity Power Broadening in a Coherently Driven Quantum-Dot-Cavity System
}

\author{
C. Roy* and S. Hughes ${ }^{\dagger}$ \\ Department of Physics, Engineering Physics and Astronomy, Queen's University, Kingston, Ontario, Canada K7L 3N6
}

(Received 2 August 2011; published 15 November 2011)

\begin{abstract}
We present a quantum optics formalism to study the intensity power broadening of a semiconductor quantum dot interacting with an acoustic-phonon bath and a high- $Q$ microcavity. Power broadening is investigated using a time-convolutionless master equation in the polaron frame, which allows for a nonperturbative treatment of the interaction of the quantum dot with the phonon reservoir. We calculate the full non-Lorentzian photoluminescence (PL) line shapes and numerically extract the intensity linewidths of the quantum-dot exciton and the cavity mode as a function of the pump rate and temperature. For increasing field strengths, multiphonon and multiphoton effects are found to be important, even for phonon-bath temperatures as low as $4 \mathrm{~K}$. We show that the interaction of the quantum dot with the phonon reservoir introduces pronounced features in the power-broadened PL line shape, enabling one to observe clear signatures of electron-phonon scattering. The PL line shapes from cavity pumping and exciton pumping are found to be distinctly different, primarily since the latter is excited through the excitonphonon reservoir. To help explain the underlying physics of phonon scattering on the power-broadened line shape, an effective phonon Lindblad master equation derived from the full time-convolutionless master equation is introduced; we identify and calculate distinct Lindblad scattering contributions from electron-phonon interactions, including effects such as excitation-induced dephasing, incoherent exciton excitation, and exciton-cavity feeding. Our effective phonon master equation is shown to reproduce the full PL intensity and the phonon-coupling effects very well, suggesting that its general Lindblad form may find widespread use in semiconductor cavity-QED.
\end{abstract}

DOI: 10.1103/PhysRevX.1.021009

Subject Areas: Optics, Quantum Physics, Semiconductor Physics

\section{INTRODUCTION}

Semiconductor quantum dots (QDs) embedded in microcavities have established themselves as a new paradigm in cavity quantum electrodynamics (cavity-QED). Technological progress in the design and fabrication of semiconductor cavity-QED systems has enabled them to be used as components in quantum information processing $[1,2]$ and for the generation of indistinguishable photons [3-6]. These quantum applications require robust cavityQED based QD devices that rest on the ability to manipulate and control the underlying quantum processes. Such quantum control is usually obtained when the cavity and QD are in the intermediate to strong-coupling regime [7-9]. Recent experimental studies have focused on the resonance fluorescence of a QD coupled to a cavity mode [10-12], and significant progress has been made in the study of an off-resonant QD-cavity system that is used to observe resonance fluorescence of a single QD [13,14]. Semiconductor micropillar systems are particularly attractive since a geometrical separation between the pump field

\footnotetext{
*chiranjeeb.roy@queensu.ca

†shughes@physics.queensu.ca
}

Published by the American Physical Society under the terms of the Creative Commons Attribution 3.0 License. Further distribution of this work must maintain attribution to the author(s) and the published article's title, journal citation, and DOI. and emitted fluorescence signal can be made, facilitating nonlinear quantum optical studies such as intensity power broadening [13].

For semiconductor cavity-QED systems, signatures of electron-acoustic-phonon scattering have been noted with incoherent excitation, resulting in off-resonant "cavity feeding" [15-22] and an asymmetric (on-resonance) vacuum Rabi doublet $[20,23,24]$. Various phonon-coupling models have been developed to try to explain these features [20,24-29]; for example, data obtained for the linear spectrum of single site-selected dots in cavities show good agreement with photon Green function theories-where the phonon coupling is included as a self-energy correction to the spectrum [22-24]. Recently, several works have also experimentally investigated coherent power (intensity) broadening in semiconductor cavity-QED systems. For example, Majumdar et al. [30] studied the role of phonon-mediated dot-cavity coupling on the powerbroadened photoluminescence (PL) intensity for a planar photonic crystal system; with experiments performed at temperatures of 30-55 K on self-assembled InAs QDs, the cavity-emitted PL intensity was found to have extraneously broadened linewidths [when compared with calculations from a simple atomic master equation (ME)]. While additional coupling may occur, e.g., from the QD to the continuum states due to the presence of the nearby wetting layer (if it exists), or due to Auger scattering [31-34], these processes are usually more important for incoherent 
excitation. For near-resonant coherent excitation, Ulhaq et al. [35] have demonstrated that dephasing and coupling due to acoustic phonons is likely the primary (and intrinsic) mechanism that couples the QD and the spectrally detuned cavity mode; their experiments were performed using self-assembled InGaAs/GaAs QDs embedded in a semiconductor micropillar cavity. While the important role of electron-phonon scattering on the linear absorption and emission spectra of self-assembled QDs is now becoming better established [36-38], there appears to be little theoretical work describing phonon effects on PL power broadening in a semiconductor cavity-QED system.

Nonlinear resonance fluorescence of an InGaAs QD embedded in a high-quality micropillar cavity was recently investigated by Ulrich et al. [39], where, in contrast to atomic cavity-QED, a clear indication of excitationinduced dephasing (EID) was found to manifest in Mollow triplet spectra with pump-induced spectral sideband broadening. Without cavity interactions, it is well known that the interaction of the driven QD with the underlying phonon reservoir can introduce additional dephasing processes and acoustic-phonon sidebands [36-38]. In a cavity system, these phonon processes can also result in significant coupling between a nonresonant cavity and a QD exciton. Very recently, a polaron ME description of phonon-induced EID in QDs and cavity-QED was described by Roy and Hughes [40]; McCutcheon and Nazir have also adopted a polaron ME approach to describe pulseexcited excitons (without cavity interactions) [41]. In light of these phonon-scattering studies and the emerging class of semiconductor cavity-QED experiments, the inclusion of phonon scattering in the theoretical description of PL power broadening in a cavity-QD system is highly desired. More generally, one requires accurate quantum optical descriptions of the semiconductor cavity-QED system, where important electron-phonon interactions are accounted for.

In this paper, we present a quantum ME formalism to study the intensity power broadening of a semiconductor cavity-QED system and identify the qualitative features of power broadening in the PL intensity introduced due to electron-phonon interactions. We exploit a timeconvolutionless ME (i.e., local in time) for the reduced density matrix of the dot-cavity subsystem, where the system-bath incoherent interaction is treated to second order $[40,41]$. The perturbative treatment is performed in the polaron frame which allows us to study the effects of phonon dephasing on the coherent part of the Hamiltonian exactly. Importantly, the cw laser driving the QD introduces additional EID effects in addition to pure dephasing due to the phonon reservoir $[40,42]$. In the appropriate limits, the model fully recovers the independent boson model (IBM) [43-45] and the Jaynes-Cummings model. The polaron transformation is particularly convenient for studying QDcavity-QED systems as it eliminates the exciton-phonon coupling and introduces a modified dot-cavity coupling and a modified radiative-decay rate $[43,45]$; in addition, there is a phonon-induced renormalization of the QD resonance frequency through the polaron shift. In the case of an exciton-driven system, the Rabi frequency of the cw laser is also renormalized by a temperature-dependent factor which essentially accounts for the dephasing of the $\mathrm{cw}$ drive due to phonon coupling. A similar polaron ME approach was previously derived by Wilson-Rae and Imamoglŭ [45], who studied the linear absorption spectrum of a cavity-QED system; their ME form [46] is nonlocal in time and is substantially more difficult to solve than the time-convolutionless form [47].

For our coherently-pumped cavity-QED investigations, we consider two distinctly different pumping scenarios: (i) the QD is driven by a coherent cw laser field, and (ii) the cavity mode is driven by a coherent $\mathrm{cw}$ laser field. We describe the generic features arising due to the relative interplay between phonon-induced dot-cavity coupling and EID in the case of a QD-driven system, and compare and contrast with power broadening for a cavity-driven system; we also discuss the differences in the integrated PL (IPL) for a dot-driven and cavity-driven system. For strong coherent drives (electromagnetic fields), the PL intensity contains significant phonon-bath signatures over a wide range of frequencies. To help explain the effects of phonon scattering in these systems, we also derive an effective phonon ME of the Lindblad form, and give simple analytical formula for the various phonon-mediated scattering rates.

Our paper is organized as follows. In Sec. II we present the model Hamiltonian and derive a time-convolutionless polaron ME where electron-phonon interactions are included to all orders. In Sec. III we introduce an effective phonon-modified Lindblad ME and compare it to the full time-convolutionless solution; the effective Lindblad ME is shown to yield good agreement with the timeconvolutionless $\mathrm{ME}$, and we use it to describe the various phonon-scattering processes. In Secs. IVA, IV B, IV C, IVD, and IVE we present and discuss our numerical results of the power-broadening line shape for both QD-driven and cavity-driven systems. In Sec. V we present our conclusions. The Appendix provides some technical details about the derivation of our effective phononscattering rates and Lindblad ME.

\section{GENERAL THEORY AND POLARON MASTER EQUATION MODEL}

The dynamics of a strongly confined QD can be modeled by considering a quantized electron-hole excitation, where the electron occupies a conduction-band state and the hole occupies a valence-band state. Neglecting quantum spin, the dominant features of a strongly confined QD can be described by the two lowest-energy bound states. This twolevel model is then conditioned by the interaction of the electrons with the lattice modes of vibration, i.e., the acoustic phonons. When the effective two-level system is driven by a cw laser field, power broadening may be 
(a)

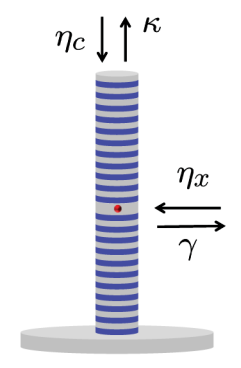

(b)
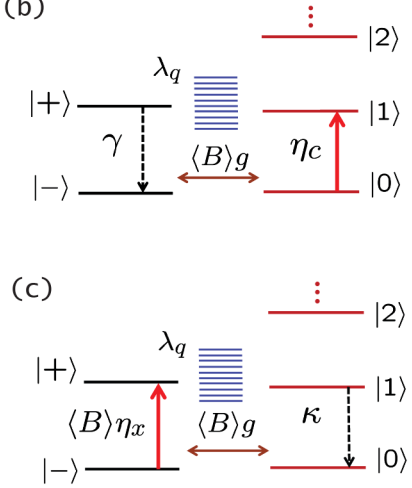

FIG. 1. (a) Schematic of an example of a semiconductor cavity used in cavity-QED (micropillar system), containing a coupled $\mathrm{QD}$, and driven by a cw laser ( $\eta_{x}$, from the side, or $\eta_{c}$, from the top). The micropillar system is advantageous to study PL power broadening since, e.g., exciton broadening can be directly studied through cavity emission-since these input-output channels are geometrically decoupled. (b) Schematic of a cavity-driven cavityQED system, where $|+\rangle$ denotes the excited QD state, $|-\rangle$ denotes the ground state, $|1\rangle$ and $|0\rangle$ represent the first excited and ground state of the cavity mode. Also shown is the phonon reservoir as blue (multiple) lines. The terms $\langle B\rangle g$ and $\langle B\rangle \eta_{x}$ represent a phonon-modified coherent reduction $(\langle B\rangle(T) \leq 1)$ in the excitoncavity coupling rate and the exciton pump rate, respectively, (see text for details). (c) Schematic of a dot-driven cavity-QED system with same energy-level notation as in (b).

substantially modified by the coupling of the QD to the phonon modes $[48,49]$. Figure 1 shows a schematic of a semiconductor cavity-QED system [Fig. 1(a)], and an energy-level diagram associated with cavity pumping [Fig. 1(b)] and exciton pumping [Fig. 1(c)]; the various parameters in the figures will be introduced below. The semiconductor cavity system of interest could be a micropillar cavity system [cf. Figure 1(a)], which allows one to excite and measure through different photon reservoirs [39] (e.g., cavity pumping and exciton emission).

Working in a frame rotating with respect to the laser pump frequency $\omega_{L}$, we first introduce the model Hamiltonian describing a cavity-QED system where the QD interacts with an acoustic-phonon reservoir:

$$
\begin{aligned}
H= & \hbar \Delta_{x L} \hat{\sigma}^{+} \hat{\sigma}^{-}+\hbar \Delta_{c L} \hat{a}^{\dagger} \hat{a}+\hbar g\left(\hat{\sigma}^{+} \hat{a}+\hat{a}^{\dagger} \hat{\sigma}^{-}\right) \\
& +H_{\text {drive }}^{x / c}+\hat{\sigma}^{+} \hat{\sigma}^{-} \sum_{q} \hbar \lambda_{q}\left(\hat{b}_{q}+\hat{b}_{q}^{\dagger}\right)+\sum_{q} \hbar \omega_{q} \hat{b}_{q}^{\dagger} \hat{b}_{q},
\end{aligned}
$$

where $\hat{b}_{q}\left(\hat{b}_{q}^{\dagger}\right)$ are the annihilation and creation operators of the phonon reservoir, $\hat{a}$ is the leaky cavity-mode annihilation operator, $\hat{\sigma}^{+}$(annihilation) and $\hat{\sigma}^{-}$(creation) are the Pauli operators of the electron-hole pair or exciton, $\Delta_{\alpha L} \equiv$ $\omega_{\alpha}-\omega_{L}(\alpha=x, c)$ are the detunings of the exciton $\left(\omega_{x}\right)$ and cavity $\left(\omega_{c}\right)$ from the coherent pump laser $\left(\omega_{L}\right)$, and $g$ is the cavity-exciton coupling strength. The pump term $H_{\text {drive }}^{x / c}$ accounts for the coherent drive on the cavity-QED

system; for a QD (exciton)-driven system, $H_{\text {drive }}^{x}=$ $\hbar \eta_{x}\left(\hat{\sigma}^{+}+\hat{\sigma}^{-}\right)$, while for a cavity-driven system, $H_{\text {drive }}^{c}=$ $\hbar \eta_{c}\left(\hat{a}+\hat{a}^{\dagger}\right)$. The defined pump rate $\eta_{x / c}$ is 2 times the classical Rabi frequency.

Transforming to the polaron frame, we eliminate the QD-phonon coupling and introduce a renormalized dot-cavity coupling strength [28]. For the case of the QD-driven system, the polaron transformation also results in a renormalized Rabi frequency, defined below. The polaron transformation $[1,43]$ can be written as

$$
H^{\prime}=\exp (S) H \exp (-S)
$$

where

$$
S=\hat{\sigma}^{+} \hat{\sigma}^{-} \sum_{q} \frac{\lambda_{q}}{\omega_{q}}\left(\hat{b}_{q}^{\dagger}-\hat{b}_{q}\right) .
$$

The transformed Hamiltonian becomes

$$
\begin{aligned}
H_{\text {sys }}^{\prime} & =\hbar\left(\Delta_{x L}-\Delta_{P}\right) \hat{\sigma}^{+} \hat{\sigma}^{-}+\hbar \Delta_{c L} \hat{a}^{\dagger} \hat{a}+\langle B\rangle \hat{X}_{g}, \\
H_{\text {bath }}^{\prime} & =\sum_{q} \hbar \omega_{q} \hat{b}_{q}^{\dagger} \hat{b}_{q}, \\
H_{\text {int }}^{\prime} & =\hat{X}_{g} \hat{\zeta}_{g}+\hat{X}_{u} \hat{\zeta}_{u},
\end{aligned}
$$

with

$$
\begin{aligned}
\hat{B}_{ \pm} & =\exp \left[ \pm \sum_{q} \frac{\lambda_{q}}{\omega_{q}}\left(\hat{b}_{q}-\hat{b}_{q}^{\dagger}\right)\right], \\
\hat{\zeta}_{g} & =\frac{1}{2}\left(\hat{B}_{+}+\hat{B}_{-}-2\langle B\rangle\right), \\
\hat{\zeta}_{u} & =\frac{1}{2 i}\left(\hat{B}_{+}-\hat{B}_{-}\right) .
\end{aligned}
$$

The polaron shift,

$$
\Delta_{P}=\int_{0}^{\infty} d \omega \frac{J(\omega)}{\omega}
$$

and the thermal average of the bath displacement operator [43],

$$
\begin{aligned}
\langle B\rangle & =\exp \left[-\frac{1}{2} \int_{0}^{\infty} d \omega \frac{J(\omega)}{\omega^{2}} \operatorname{coth}(\beta \hbar \omega / 2)\right], \\
& =\exp \left[-\frac{1}{2} \sum_{q}\left(\frac{\lambda_{q}}{\omega_{q}}\right)^{2}\left(2 \bar{n}_{q}+1\right)\right], \\
& =\left\langle B_{+}\right\rangle=\left\langle B_{-}\right\rangle,
\end{aligned}
$$

where $\bar{n}_{q} \equiv\left\langle\hat{b}_{q}^{\dagger} \hat{b}_{q}\right\rangle=\left[e^{\beta \hbar \omega_{q}}-1\right]^{-1}$ is the mean phonon occupation number (Bose-Einstein distribution) at a bath temperature, $T=1 / K b \beta$, and $J(\omega)$ is the phonon spectral function defined later in Eq. (14). For clarity, we will henceforth assume that the polaron shift is implicitly included in our definition of $\omega_{x}$ (one should, however, keep in mind that this shift is temperature dependent). For a dot (exciton)-driven system, $\hat{X}_{g}$ and $\hat{X}_{u}$ are defined through

$$
\begin{aligned}
& \hat{X}_{g}=\hbar g\left(\hat{a}^{\dagger} \hat{\sigma}^{-}+\hat{\sigma}^{+} \hat{a}\right)+\hbar \eta_{x}\left(\hat{\sigma}^{-}+\hat{\sigma}^{+}\right), \\
& \hat{X}_{u}=i \hbar\left[g\left(\hat{\sigma}^{+} \hat{a}-\hat{a}^{\dagger} \hat{\sigma}^{-}\right)+i \hbar \eta_{x}\left(\hat{\sigma}^{+}-\hat{\sigma}^{-}\right)\right],
\end{aligned}
$$

and for a cavity-driven system, 


$$
\begin{aligned}
& \hat{X}_{g}=\hbar g\left(\hat{a}^{\dagger} \hat{\sigma}^{-}+\hat{\sigma}^{+} \hat{a}\right), \\
& \hat{X}_{u}=i \hbar g\left(\hat{\sigma}^{+} \hat{a}-\hat{a}^{\dagger} \hat{\sigma}^{-}\right) .
\end{aligned}
$$

It is worth noting the slightly unusual definition of the system Hamiltonian, Eq. (4a). The usual (but in general, incorrect) decomposition of the system Hamiltonian to include only the noninteracting QD and cavity parts does not take into account the effect of the coherent $\mathrm{cw}$ drive on the system Hamiltonian. As the cavity and the QD systems are internally coupled, as discussed by Carmichael and Walls [50], this leads to violation of detailed balance. The system Hamiltonian written above leads to the correct form of the density operator while preserving detailed balance. Moreover, it includes the effect of dot-cavity coupling and the dot-cw driving on the coherent part of the Hamiltonian to all orders.

Next, we unitarily transform to a frame of reference defined by this system Hamiltonian, which we will use to obtain a time-convolutionless ME; the net effect of this transform, e.g., in the case of resonance fluorescence with an exciton-driven system, results in Mollow triplet peaks that sample the asymmetric phonon bath at the dressed eigenfrequencies - as determined by the modified system Hamiltonian. Somewhat similar techniques (i.e., bath sampling at the dressed resonances) have been employed to study atomic dynamics in generalized (engineered) photon reservoirs, including photonic-band gap materials [51] and squeezed reservoirs [52].

Phenomenologically, we include the radiative decay of the QD and the cavity mode decay as Liouvillian superoperators acting on the reduced density matrix [20]. In addition, we incorporate an additional pure-dephasing process beyond the IBM with a rate $\gamma^{\prime}$-this accounts for the broadening of the zero-phonon line (ZPL) with increasing temperatures [36,53-61]. Though there is some controversy about what causes the broadening of the ZPL, e.g., spectral diffusion, anharmonicity effects, [57,58] phonon scattering from interfaces, $[59,60]$, and a modified phonon spectrum [61], it is well known that the ZPL broadens as a function of temperature with a Lorentzian scattering process; thus, we treat the broadening of the ZPL phenomenologically, while accounting for broadening as a function of temperature similar to experiments $[20,53,56]$, with $\gamma^{\prime}(T)$ scaling as $\sim 1 \mu \mathrm{eV} / \mathrm{K}$. The various Lindblad superoperators act on the reduced system density matrix, and are defined through

$$
\begin{aligned}
L(\rho)= & \frac{\tilde{\gamma}}{2}\left(2 \hat{\sigma}^{-} \rho \hat{\sigma}^{+}-\hat{\sigma}^{+} \hat{\sigma}^{-} \rho-\rho \hat{\sigma}^{+} \hat{\sigma}^{-}\right) \\
& +\kappa\left(2 \hat{a} \rho \hat{a}^{\dagger}-\hat{a}^{\dagger} \hat{a} \rho-\rho \hat{a}^{\dagger} \hat{a}\right) \\
& +\frac{\gamma^{\prime}}{2}\left(2 \hat{\sigma}_{11} \rho \hat{\sigma}_{11}-\hat{\sigma}_{11} \hat{\sigma}_{11} \rho-\rho \hat{\sigma}_{11} \hat{\sigma}_{11}\right),
\end{aligned}
$$

where $2 \kappa$ is the cavity decay rate, $\tilde{\gamma}=\gamma\langle B\rangle^{2}$ is the radiative-decay rate, and $\hat{\sigma}_{11}=\hat{\sigma}^{+} \hat{\sigma}^{-}$. The radiativedecay rate has an additional renormalization by a factor of $\langle B\rangle^{2}$, which reduces the effective radiative-decay rate in the presence of phonons [62].

We then derive a time-convolutionless ME for the reduced density operator $\rho(t)$ of the cavity-QED system [41] in the second-order Born approximation (for incoherent bath coupling). The time-convolutionless form of the ME, though local in time, is known to capture non-Markov effects due to the reservoir [63]. However, for our analysis, we will make a Markov approximation as typical phonon processes are substantially faster (i.e., a few picoseconds) than the relevant system dynamics by at least an order of magnitude. This allows us to obtain effective rates which naturally depend on the spectral densities of the phonon spectral function that are locally sampled by the dressed resonances. We have checked that the Markov limit of the fully non-Markovian time-convolutionless $\mathrm{ME}$ is rigorously valid for the system and excitation (cw) cases of interest. Thus, while it is straightforward to carry out nonMarkov calculations, it is not necessary here-they give identical results.

In the interaction picture described by $H_{\text {sys }}^{\prime}$, we consider the exciton-photon-phonon coupling $H_{\text {int }}^{\prime}$ to second order (Born approximation), and trace over the phonon degrees of freedom to obtain a Markovian time-convolutionless ME [40,41]:

$$
\begin{aligned}
\frac{\partial \rho(t)}{\partial t}= & \frac{1}{i \hbar}\left[H_{\mathrm{sys}}^{\prime}, \rho(t)\right]+L(\rho)-\frac{1}{\hbar^{2}} \int_{0}^{\infty} d \tau \sum_{m=g, u}\left\{G_{m}(\tau)\right. \\
& \left.\times\left[\hat{X}_{m}, e^{-i H_{\mathrm{sys}}^{\prime} \tau / \hbar} \hat{X}_{m} e^{i H_{\mathrm{sys}}^{\prime} \tau / \hbar} \rho(t)\right]+\text { H.c. }\right\},
\end{aligned}
$$

where $G_{g / u}(t)=\left\langle\zeta_{g / u}(t) \zeta_{g / u}(0)\right\rangle$, and we have assumed that the phonon bath is in thermal equilibrium. The polaron Green functions are $[43,45]$

$$
\begin{aligned}
G_{g}(t) & =\langle B\rangle^{2}\{\cosh [\phi(t)]-1\}, \\
G_{u}(t) & =\langle B\rangle^{2} \sinh [\phi(t)],
\end{aligned}
$$

which depend on the phonon correlation function,

$$
\begin{aligned}
\phi(t) & =\int_{0}^{\infty} d \omega \frac{J(\omega)}{\omega^{2}}[\operatorname{coth}(\beta \hbar \omega / 2) \cos (\omega t)-i \sin (\omega t)], \\
& =\sum_{q}\left(\frac{\lambda_{q}}{\omega_{q}}\right)^{2}\left[\left(\bar{n}_{q}+1\right) e^{-i \omega_{q} t}+\bar{n}_{q} e^{i \omega_{q} t}\right],
\end{aligned}
$$

where $J(\omega)$ is the characteristic phonon spectral function, defined in this work as

$$
J(\omega)=\alpha_{p} \omega^{3} \exp \left(-\frac{\omega^{2}}{2 \omega_{b}^{2}}\right) .
$$

This form of the spectral function [Eq. (14)] describes the interaction between the electrons and the longitudinal acoustic phonons via a deformation potential coupling which is the main source of dephasing in self-assembled 
InAs/GaAs QDs. For all our calculations that follow, we use parameters suitable for InAs/GaAs QDs [64], with $\omega_{b}=1 \mathrm{meV}\left(\omega_{b}\right.$ is a high frequency cutoff proportional to the inverse of the typical electronic localization length in the QD) and $\alpha_{p} /(2 \pi)^{2}=0.06 \mathrm{ps}^{2}$; these values vary somewhat in the literature, though we have taken ours from fitting recent experiments $[24,39,40]$. Using the parameters above, e.g., at $T=10 \mathrm{~K}$, yields a polaron shift, $\Delta_{P} \equiv 42 \mu \mathrm{eV}$, and a Franck-Condon renormalization, $\langle B\rangle=0.84$. With these phonon parameters, we already see that clearly the coherent renormalization effects will be important for anlyzing PL intensity for QD-cavity systems, even at relatively low phonon-bath temperatures.

We briefly mention that there are other electronphonon-scattering models that can go beyond the polaron ME approach. For example, McCutcheon et al. [65] recently introduced a more general ME technique to describe the nonequilibrium dynamics of a QD system interacting with a phonon reservoir based on a variational formulation (with no cavity coupling). This elegant approach extends the validity of the ME to parameter regimes, $\eta_{x} \geq \omega_{b}$, where the $\mathrm{ME}$ in the polaron frame can break down. However, the pump parameter regimes that we study in this work $\left(\eta_{x / c} \ll \omega_{b}\right)$ are well within the domain of validity of our ME, so we can safely use the polaron ME, while also accounting for cavity coupling [40]. A more general description of the system dynamics valid in all regimes can be obtained using a quasiadiabatic pathintegral approach [66,67]. The benefits of our polaron $\mathrm{ME}$ is that the solution, even with multiphonon and multiphoton effects included, is relatively straightforward, and it has already been used to help explain experiments for coherently excited dots in the regime of cavity-QED $[39,40]$. Moreover, as we will show below, one can derive a user-friendly Lindblad ME that contains many of the key features of phonon interactions in semiconductor cavity-QED systems.

For numerical calculations, we solve the above ME with steady-state pumping (i.e., $\eta_{x / c}$ are time-independent), with the exciton initially in the ground state. Prior to these dynamical calculations, we compute the phonon-scattering terms in Eq. (11), whose solution is naturally problem dependent (through $H_{\text {sys }}^{\prime}$ ). Thus, there are no fixed phonon-scattering rates for analyzing QD power broadening as a function of pump power, as the phonon-scattering rates are pump dependent. The same arguments apply for studying power broadening as a function of temperature; one must obtain the phonon-induced scattering rates for each pump value and temperature. Experimentally, the PL intensity line shape is usually obtained by measuring the QD exciton intensity $\left(I_{x}\right)$ or cavity-mode intensity $\left(I_{c}\right)$ as a function of an increasing pump field. To connect to these quantities, we solve the above ME in a Jaynes-Cummings basis with states $|0\rangle,|1 L\rangle,|1 U\rangle,|2 L\rangle,|2 U\rangle, \ldots$, and compute the steady-state exciton and cavity-photon populations, $\left.\bar{n}_{x} \equiv\left\langle\sigma^{+} \sigma^{-}\right\rangle\right|_{s s} \propto I_{x}$ and $\left.\bar{n}_{c} \equiv\left\langle a^{\dagger} a\right\rangle\right|_{s s} \propto I_{c}$. Defining the photon states $|n\rangle$, with $n=0,1,2, \ldots$, and exciton states $|+|-\rangle$, the Jaynes-Cummings ladder states are then related to the bare states, e.g., through $|0\rangle=|-\rangle|0\rangle,|1 L\rangle=$ $\frac{1}{\sqrt{2}}(|-\rangle|1\rangle-|+\rangle|0\rangle),|1 U\rangle=\frac{1}{\sqrt{2}}(|-\rangle|1\rangle+|+\rangle|0\rangle)$, etc.

In our calculations we make use of the quantum optics toolbox by Tan [68], and find that a basis truncation to twophoton correlations (two photons or 5 states) is sufficient and/or necessary for all the dot-driven simulations, while a basis truncation to six photon correlations (six photons or 13 states) is sufficient and/or necessary for all cavity calculations that follow. The role of multiphoton effects depends on the value of the dot-cavity coupling rate $g$, which we choose to be $g=20 \mu \mathrm{eV}$, which is consistent with typical semiconductor cavity-QED power-broadening experiments (e.g., see Refs. [30,35]). A detailed discussion of the role of multiphoton, and multiphonon processes, is presented in Sec. IV B.

\section{EFFECTIVE PHONON MASTER EQUATION OF THE LINDBLAD FORM}

Our polaron ME [Eq. (11)] includes both coherent and incoherent contributions from electron-phonon scattering, but some care and insight is needed in extracting the relevant incoherent scattering rates. It is therefore instructive to construct a simplified phonon-modified ME of the Lindblad form, which we call an effective phonon master equation (EPME); we do this by simplifying the term, $e^{-i H_{\mathrm{sys}}^{\prime} \tau / \hbar} \hat{X}_{m} e^{i H_{\mathrm{sy}}^{\prime} \tau / \hbar}$, appearing in the full timeconvolutionless ME [Eq. (11)]. The resulting Lindbladform $\mathrm{ME}$ enables a very simple numerical solution and facilitates the extraction of various phonon-induced scattering rates in a clear and transparent way. We expect that the integral in Eq. (11) can be approximated, under certain circumstances, by only including the phase evolution of the operators $\hat{X}_{g, u}$ with respect to the noninteracting part of the system evolution. Further, for a QD-driven system we only include terms proportional to $g^{2}$ and $\eta_{x}^{2}$ and ignore cross terms proportional to $g \eta_{x}$; the inclusion of the cross terms do not preserve the Lindblad form and contribute very little to the overall broadening line shape as can be demonstrated numerically. For a cavity-driven system, we again include the phase evolution of the operators $\hat{X}_{g, u}$ with respect to the noninteracting part of the system evolution; however, the effective Lindblad description has only contributions which are proportional to $g^{2}$ - since $\hat{X}_{g, u}$ do not depend on $\eta_{c}$. We will, of course, compare the EPME solution with the full numerical solution of the polaron time-convolutionless ME, i.e., Eq. (11); the prime purpose of the EPME is to help elucidate the physics of phononinduced incoherent scattering, though we will highlight regimes where it can work quite well in accurately describing the full characteristics of the entire power-broadened PL line shape. 
We postulate that the dynamics of the QD-driven system can now be approximately described through

$$
\frac{\partial \rho(t)}{\partial t}=\frac{1}{i \hbar}\left[H_{\mathrm{sys}}^{\mathrm{eff}}, \rho(t)\right]+L(\rho)+L_{\mathrm{ph}}(\rho),
$$

where $L_{\mathrm{ph}}(\rho)$ (ph refers to phonon) is given by

$$
\begin{aligned}
L_{\mathrm{ph}}(\rho)= & \frac{\Gamma_{\mathrm{ph}}^{\sigma^{-}}}{2} L\left(\hat{\sigma}^{-}\right)+\frac{\Gamma_{\mathrm{ph}}^{\sigma^{+}}}{2} L\left(\hat{\sigma}^{+}\right)+\frac{\Gamma_{\mathrm{ph}}^{\sigma^{+} a}}{2} L\left(\hat{\sigma}^{+} \hat{a}\right) \\
& +\frac{\Gamma_{\mathrm{ph}}^{a^{\dagger} \sigma^{-}}}{2} L\left(\hat{a}^{\dagger} \hat{\sigma}^{-}\right),
\end{aligned}
$$

and the superoperator $L(\hat{D})$ is defined as

$$
L(\hat{D})=2 \hat{D} \rho \hat{D}^{\dagger}-\hat{D}^{\dagger} \hat{D} \rho-\rho \hat{D}^{\dagger} \hat{D} .
$$

The above effective ME [Eq. (15)] has a remarkably simple form, and its general format should be familiar to many researchers who have been using atomic cavity-QED models to connect to experimental data with semiconductor cavity-QED systems. However, it must be used with caution, as it is only valid within certain regimes where the above noted approximations are good. The phononmediated rates, which drive the effective Lindblad dynamics, are derived to be (see the Appendix)

$$
\begin{gathered}
\Gamma_{\mathrm{ph}}^{\sigma^{-} / \sigma^{+}}=2\langle B\rangle^{2} \eta_{x}^{2} \operatorname{Re}\left[\int_{0}^{\infty} d \tau e^{ \pm i \Delta_{x L} \tau}\left(e^{\phi(\tau)}-1\right)\right], \\
\Gamma_{\mathrm{ph}}^{\sigma^{+} a / a^{\dagger} \sigma^{-}}=2\langle B\rangle^{2} g^{2} \operatorname{Re}\left[\int_{0}^{\infty} d \tau e^{ \pm i \Delta_{c x} \tau}\left(e^{\phi(\tau)}-1\right)\right],
\end{gathered}
$$

where $\Delta_{c x}=\omega_{c}-\omega_{x}$ is the cavity-exciton detuning. Figure 2 shows a schematic of the various effective phonon-scattering processes: $\Gamma_{\mathrm{ph}}^{\sigma^{+}}$describes phononassisted incoherent excitation and EID (pump-induced broadening), $\Gamma_{\mathrm{ph}}^{\sigma^{-}}$describes enhanced radiative decay and EID, $\Gamma_{\mathrm{ph}}^{\sigma^{+} a}$ describes (the somewhat unlikely scenario of) exciton excitation via the emission of a cavity photon, and $\Gamma_{\mathrm{ph}}^{a^{\dagger} \sigma^{-}}$describes the process of cavity excitation (cavity feeding) via the absorption of a photon. Importantly, all of these scattering events are driven by electron-phonon interactions and they cause effects that are significantly different to simple pure-dephasing models. In fact, pure dephasing through $\gamma^{\prime}$ only results in Lorentzian coupling, and is found to play a minor role in what follows below.

Our formalism above shows that for a cavity-driven system, $\Gamma_{\mathrm{ph}}^{\sigma^{+} / \sigma^{-}}=0$ and $\Delta_{\mathrm{ph}}^{\sigma^{-} / \sigma^{+}}=0$, and there is no phonon-induced EID due to the lack of any coupling of the drive with the phonon reservoir. We therefore expect (and find) substantially different intensity power broadening between dot-driven and cavity-driven systems; both of these exciton-driven and cavity-driven models are also markedly different from simple atomic models. We highlight that a similar exciton-cavity (feeding) rate $\Gamma_{\mathrm{ph}}^{a^{\dagger} \sigma^{-}}$has been derived by Xue et al. [25] and by Hohenester [27], though these were (a) $\Gamma_{\mathrm{ph}}^{\sigma^{+}}$

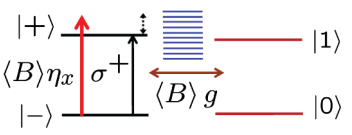

(c) $\Gamma_{\mathrm{ph}}^{\sigma^{+} a}$

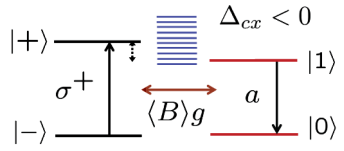

(b) $\Gamma_{\mathrm{ph}}^{\sigma^{-}}$

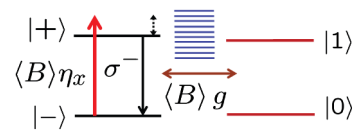

(d) $\Gamma_{\mathrm{ph}}^{a^{\dagger} \sigma}$

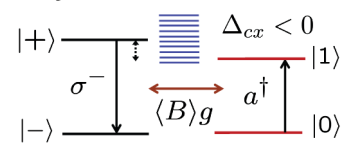

FIG. 2. Schematic of the phonon-scattering processes: (a) $\Gamma_{\mathrm{ph}}^{\sigma^{+}}$, (b) $\Gamma_{\mathrm{ph}}^{\sigma^{-}}$, (c) $\Gamma_{\mathrm{ph}}^{\sigma^{+} a}$, and (d) $\Gamma_{\mathrm{ph}}^{a^{\dagger} \sigma^{-}}$, for a QD-driven system introduced in the effective Lindblad description and defined in Eqs. (18) and (19). The various symbols are the same as in Fig. 1. Processes (a) and (b) describe the phonon-induced incoherent excitation and excitation-induced dephasing; process (c) describes phonon-induced exciton excitation via cavity decay, though this process has a small probability because of a relatively large cavity decay rate $(\kappa)$; process (d) describes phononinduced cavity excitation through the decay of an exciton; at low temperature this (cavity-feeding) process occurs primarily via phonon emission, and will be more efficient for a cavity mode that is red shifted with respect to the exciton. The sign of the cavity detuning in (c)-(d) is just an example, and note that the phonon interactions with $\Delta_{c x}=\Delta$ or $\Delta_{c x}=-\Delta$ will be different (especially at lower temperatures).

obtained for an undriven cavity-QED system. Both of these useful approaches also use a polaron frame to describe the incoherent scattering, though the end equations have some potential problems for small cavity-exciton detunings (where, admittedly, these effective rates are at best approximate anyway), and neither approach includes a coherent (temperature-dependent) reduction in $g \rightarrow\langle B\rangle g$. For example, Hohenester [27] derives the following exciton-cavity feeding rate: $\Gamma_{\mathrm{ph}}^{a^{\dagger} \sigma^{-}}=2 g^{2} \operatorname{Re}\left[\int_{0}^{\infty} d \tau e^{-i \Delta_{c x} \tau} e^{-\phi(\tau)}\right]$, which has a similar form to our Eq. (19) (apart from the sign of the phase and the need to subtract off a background term for small detuning), but does not include $\langle B(\mathrm{~T})\rangle^{2}$-an important temperature-dependent term. The general predictions using this rate formula are consistent with experiments [26], and all these aforementioned polaron formalisms produce qualitatively the same trend as a function of cavity-exciton detuning (compare results in Refs. [25,27] with those in Figs. 5 and 6).

An alternative effective Lindblad ME with the same process identified in Refs. [25,27] and above (i.e., for exciton-cavity coupling), was recently presented by Majumdar et al. [14] and used in part to study the role of phonon scattering for the cavity-emitted resonance fluorescence spectrum. The influence of phonons was included as two additional incoherent decay terms, which were included to second order in the QD-phonon coupling. Unlike the polaronic approaches above, electron-phonon interactions were included only to first order, which is 
generally not valid in these cavity-QED systems - even at low temperature [40] (see Sec. IV B). Potentially more problematic is the fact that the effects of the coherent drive on the phonon reservoir and the associated EID effects are missing; in contrast, we find these to be the dominant source of broadening from electron-phonon scattering. The need for EID processes in the coherently driven semiconductor cavity-QED system has already been shown for the Mollow triplet, both experimentally [39] and theoretically [40]. However, for a cavity-driven system, the phonon-scattering process described through $L_{\mathrm{ph}}^{a^{\dagger} \sigma^{-}}$is the main effect of phonon coupling, and this process is partially captured (through weak phonon interactions) in Ref. [14].

In addition to the phonon-induced Lindblad decay rates above, one also has phonon-mediated frequency shifts beyond the polaron shift. The effective Hamiltonian, describing the coherent part of the system evolution $H_{\text {sys }}^{\text {eff }}$ becomes

$$
\begin{aligned}
H_{\mathrm{sys}}^{\mathrm{eff}}= & \hbar \Delta_{x L} \hat{\sigma}^{+} \hat{\sigma}^{-}+\hbar \Delta_{c L} \hat{a}^{\dagger} \hat{a}+\langle B\rangle \hat{X}_{g} \\
& +\hbar \Delta_{\mathrm{ph}}^{\sigma^{+} a} \hat{a}^{\dagger} \hat{\sigma}^{-} \hat{\sigma}^{+} \hat{a}+\hbar \Delta_{\mathrm{ph}}^{a^{\dagger} \sigma^{-}} \hat{\sigma}^{+} \hat{a} \hat{a}^{\dagger} \hat{\sigma}^{-} \\
& +\hbar \Delta_{\mathrm{ph}}^{\sigma^{-}} \hat{\sigma}^{-} \hat{\sigma}^{+}+\hbar \Delta_{\mathrm{ph}}^{\sigma^{+}} \hat{\sigma}^{+} \hat{\sigma}^{-},
\end{aligned}
$$

with

$$
\begin{gathered}
\Delta_{\mathrm{ph}}^{\sigma^{-} / \sigma^{+}}=\langle B\rangle^{2} \eta_{x}^{2} \operatorname{Im}\left\{\int_{0}^{\infty} d \tau e^{ \pm i \Delta_{x L} \tau}\left[e^{\phi(\tau)}-1\right]\right\}, \\
\Delta_{\mathrm{ph}}^{\sigma^{+} a / a^{\dagger} \sigma^{-}}=\langle B\rangle^{2} g^{2} \operatorname{Im}\left\{\int_{0}^{\infty} d \tau e^{ \pm i \Delta_{c x} \tau}\left[e^{\phi(\tau)}-1\right]\right\},
\end{gathered}
$$

where $\Delta_{\mathrm{ph}}^{\sigma^{+} a}, \Delta_{\mathrm{ph}}^{a^{\dagger} \sigma^{-}}, \Delta_{\mathrm{ph}}^{\sigma^{+}}$, and $\Delta_{\mathrm{ph}}^{\sigma^{-}}$are the Stark shifts (which scale proportionally with $\langle B\rangle g^{2}$ or $\langle B\rangle \eta_{x}^{2}$ ).

\section{NUMERICAL RESULTS}

\section{A. Role of phonon scattering on intensity power broadening: Effective phonon ME versus the full time-convolutionless ME}

We first investigate the role of the four phonon Lindblad terms in a typical power-broadened PL intensity computed with our EPME [Eq. (15)] and compare with the full solution [Eq. (11): time-convolutionless ME]. The main parameters are listed in Fig. 3, and we have adopted system parameters and coupling constants similar to those in recent semiconductor experiments [30,35].

In Fig. 3 we study the role of $\Gamma_{\mathrm{ph}}^{\sigma^{-}}, \Gamma_{\mathrm{ph}}^{\sigma^{+}}, \Gamma_{\mathrm{ph}}^{a^{\dagger} \sigma^{-}}$, and $\Gamma_{\mathrm{ph}}^{\sigma^{+} a}$, as defined in Eqs. (18) and (19), on the power-broadening line shape $\left(I_{c} \propto \bar{n}_{c}\right)$; here we use $\eta_{x}=40 \mu \mathrm{eV}$ for a QD-driven system at a bath temperature of $T=4 \mathrm{~K}$, and study two different cavity-exciton detunings, (a), (b) $\Delta_{c x}=3 \mathrm{meV}$ and (c), (d) $\Delta_{c x}=-3 \mathrm{meV}$. The corresponding peak $\bar{n}_{c}$ that results from this interaction is around $4 \times 10^{-5}$. To better highlight the various scattering
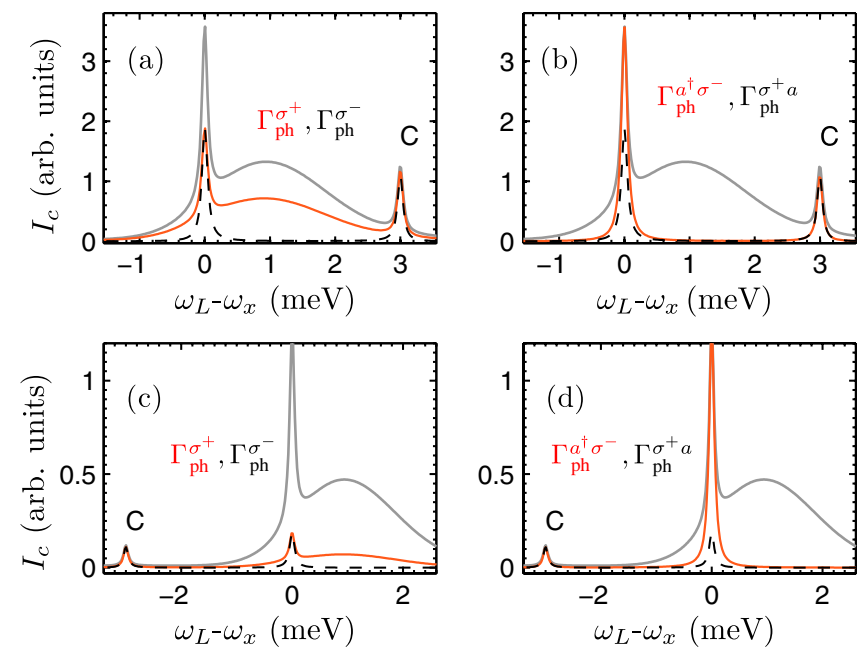

FIG. 3. (a) Influence of the effective phonon-scattering rates, $\Gamma_{\mathrm{ph}}^{\sigma^{+}}$(red line) and $\Gamma_{\mathrm{ph}}^{\sigma^{-}}$(dashed line) [defined in Eq. (18)], on the PL intensity $I_{c}$ for a QD-driven system at $T=4 \mathrm{~K}$ with $\Delta_{c x}=$ $3 \mathrm{meV}$. Also shown is the full polaron ME solution (grey line) [Eq. (11)]. We plot the cavity intensity $\left(I_{c}\right)$ for exciton excitation as a function of QD-laser detuning and show the contribution of one effective Lindblad rate per calculation; the collective influence of these processes is shown later in Fig. 5 and compared with the full solution. (b) As in (a), but for effective phononscattering rates, $\Gamma_{\mathrm{ph}}^{a^{\dagger} \sigma^{-}}$(red line) and $\Gamma_{\mathrm{ph}}^{\sigma^{+} a}$ (dashed line) [defined in Eq. (19)]. The system and material parameters are $\gamma=$ $2 \mu \mathrm{eV}, \kappa=50 \mu \mathrm{eV}, g=20 \mu \mathrm{eV}, \eta_{x}=40 \mu \mathrm{eV}, \gamma^{\prime}(4 \mathrm{~K})=$ $2 \mu \mathrm{eV}$, and we compute $\langle B\rangle(4 \mathrm{~K})=0.91$. (c)-(d) As in (a)-(b) but with $\Delta_{c x}=-3 \mathrm{meV}$.

mechanisms, we include only one of the Lindblad terms in each calculation, as labeled in the plots. By looking at Figs. 3(a) and 3(c), it is clear that the process $L\left(\hat{\sigma}^{+}\right)$is primarily responsible for incoherently exciting the phonon
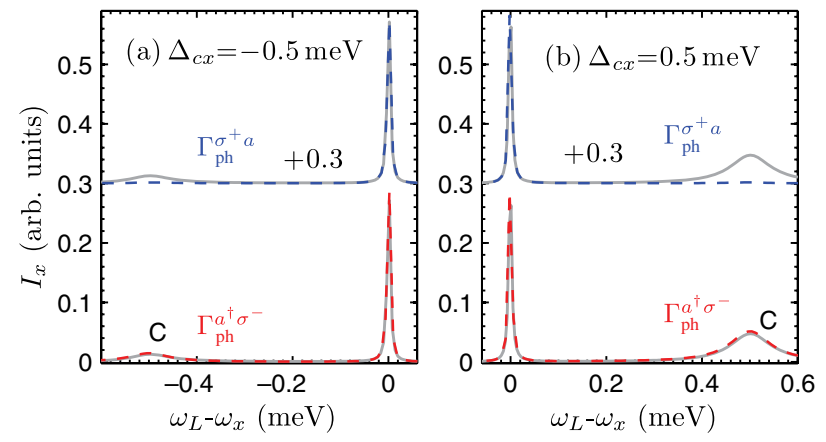

FIG. 4. (a) Influence of the role of the effective phonon-scattering rates, $\Gamma_{\mathrm{ph}}^{a^{\dagger} \sigma^{-}}$(red dashed line) and $\Gamma_{\mathrm{ph}}^{\sigma^{+} a}$ (blue dashed line), on the PL intensity $I_{x}$ for a cavity-driven system at $T=4 \mathrm{~K}$ for $\Delta_{c x}=$ $-0.5 \mathrm{meV}$. Also shown is the full polaron ME solution (grey lines) [Eq. (11)]. For clarity, the $\Gamma_{\mathrm{ph}}^{\sigma^{+} a}$ curves are shifted vertically by 0.3 , along with the full polaron ME solution. We plot the contribution of one effective Lindblad rate per calculation. (b) As in (a) but for $\Delta_{c x}=0.5 \mathrm{meV}$. The parameters are the same as in Fig. 3, but with $\eta_{c}=40 \mu \mathrm{eV}$ (cavity pumping). Since we have cavity excitation, then the phonon-scattering rates $\sigma_{\mathrm{ph}}^{\sigma^{+}} / \sigma^{-}$are zero. 
sidebands [cf. 3(a)] and EID; while process $L\left(\hat{\sigma}^{-}\right)$introduces further pump-dependent EID, as will be highlighted in detail later (note that this process has the same Lindblad operator terms as $\gamma$ or $\tilde{\gamma}$ ). The broad background centered at $\omega_{L}-\omega_{x} \approx 1 \mathrm{meV}$ is present only with $\Gamma^{\sigma^{+}}$scattering included. The exciton-cavity scattering processes, $L\left(\hat{a}^{\dagger} \hat{\sigma}^{-}\right)$and $L\left(\hat{\sigma}^{+} \hat{a}\right)$, account for cavity excitation and cavity destruction, respectively, by phonon-assisted processes and these affect the relative magnitudes of the cavity-measured PL intensity at different temperatures and drives. Figures $3(\mathrm{~b})$ and $3(\mathrm{~d})$ demonstrate that $L\left(\hat{a}^{\dagger} \hat{\sigma}^{-}\right)$is the main cavity-exciton coupling (feeding) term; this mechanism results in enhanced cavity-photon numbers at the exciton transition, especially when the cavity is red shifted from the exciton-since phonon emission is favorable at lower temperatures. In contrast, the $L\left(\hat{\sigma}^{+} \hat{a}\right)$ process gives no noticeable exciton-cavity coupling because of the (relatively) large cavity decay rate [27].

In Fig. 4 we carry out a similar exercise for a cavityexcited system $\left(\eta_{c}=40 \mu \mathrm{eV}\right)$, calculating $I_{x}\left(\propto \bar{n}_{x}\right)$, where we study the influence of $L\left(\hat{a}^{\dagger} \hat{\sigma}^{-}\right)$and $L\left(\hat{\sigma}^{+} \hat{a}\right)$ on power broadening; here we find excellent agreement with only the $\Gamma_{\mathrm{ph}}^{a^{\dagger} \sigma^{-}}$scattering term (lower red, dashed curve) compared to the full ME solution (lower grey,
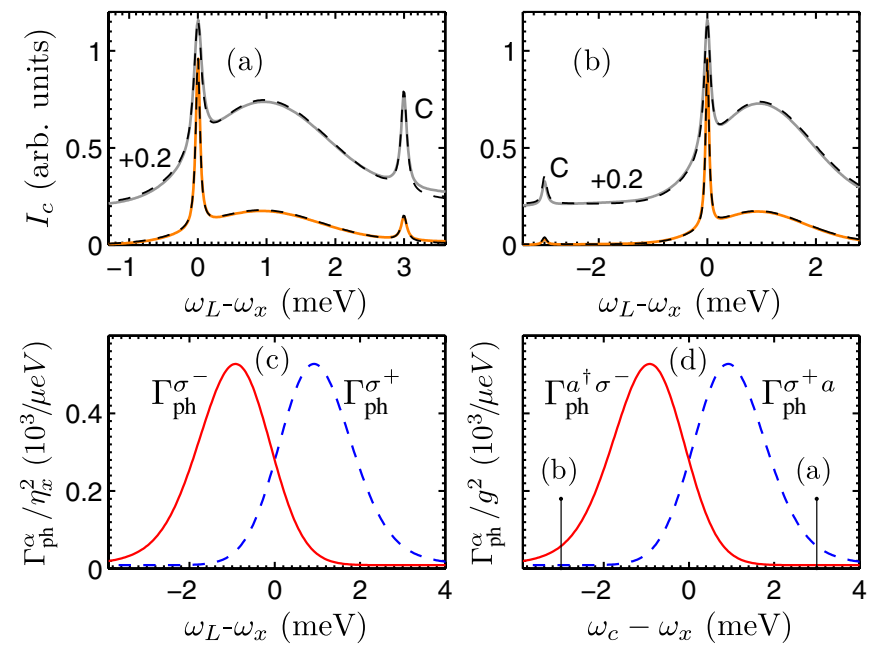

FIG. 5. Phonon bath at $T=4 \mathrm{~K}$. (a)-(b) Normalized cavitymode intensity $\left(I_{c}\right)$ for a dot-driven system as a function of QD-laser detuning for two different dot-cavity detunings, (a) $\Delta_{c x}=3 \mathrm{meV}$ and (b) $\Delta_{c x}=-3 \mathrm{meV}$, and for two different values of the cw laser Rabi frequency (orange line, corresponds to $\eta_{x}=20 \mu \mathrm{eV}$, and grey, upper solid curve, corresponds to $\eta_{x}=40 \mu \mathrm{eV}$ ). Also shown (black dashed lines) is the PL intensity obtained using the effective Lindblad form of the full time-convolutionless ME. Note that we have vertically shifted the PL intensity for $\eta_{x}=40 \mu \mathrm{eV}$ by 0.2 for clarity. (c) Plot of the phonon rates $\Gamma_{\mathrm{ph}}^{\sigma^{+} / \sigma^{-}}$(see text) for $\Delta_{c x}=-3 \mathrm{meV}$ as a function of QD-laser detuning. Note $\Gamma_{\mathrm{ph}}^{\sigma^{-}}\left(-\Delta_{c x}\right)=\Gamma_{\mathrm{ph}}^{\sigma^{+}}\left(\Delta_{c x}\right)$. (d) Plot of the phonon rates $\Gamma_{\mathrm{ph}}^{\sigma^{+} a / a^{\dagger} \sigma^{-}}$as a function of QD-cavity detuning. For convenience, the scattering rates are plotted in normalized units with respect to $\eta_{x}^{2}$ and $g^{2}$. solid line), which results in a significant exciton-cavity feeding process via phonon emission [cavity is red detuned in (a) cf. Fig. 2(d)]. Again we find that $L\left(\hat{\sigma}^{+} \hat{a}\right)$ gives no noticeable cavity feeding. For this cavity-driven system, we have chosen $\Delta_{c x}= \pm 0.5 \mathrm{meV}$ instead of $\Delta_{c x}=$ $\pm 3 \mathrm{meV}$ (which we chose earlier for the exciton-driven system); this is because a strong exciton-driven system invariably kicks up the phonon sidebands even at low temperatures that can swamp the emission at the cavity mode; so we use a larger cavity-exciton detuning for the exciton-driven case. The cavity-driven system is thus much cleaner to analyze for smaller cavity-exciton detunings, and the exciton-measured PL intensity is also substantially reduced for larger detunings. We remark that the corresponding peak $\bar{n}_{x}$ for this excitation regime is around 0.3.

In Figs. 5(a) and 5(b) we plot the normalized cavitymode intensity, $I_{c} \propto \bar{n}_{c}$, for a dot-driven system as a function of QD-laser detuning, again for the two different cavity-exciton detunings, (a) $\Delta_{c x}=3 \mathrm{meV}$ and (b) $\Delta_{c x}=$ $-3 \mathrm{meV}$, at $T=4 \mathrm{~K}$. We also show the PL intensity for $\eta_{x}=20 \mu \mathrm{eV}$ and $\eta_{x}=40 \mu \mathrm{eV}$. To study the effects of increasing temperatures, in Fig. 6 we plot the $I_{c}$ at $T=$ $20 \mathrm{~K}$. In these graphs, we show the total power-broadened PL intensity obtained using the EPME [dashed lines in (a), (b)] and compare with the full polaron ME solution [solid lines in (a), (b)]; given the approximations made in the derivation of the EPME, the agreement is remarkably good. The frequency-shift terms $\Delta_{\mathrm{ph}}^{\alpha}$ are found to be very small here and can be neglected for the cases shown. Note that the phenomenological pure-dephasing rates are temperature dependent, where we choose $\gamma^{\prime}(4 \mathrm{~K})=2 \mu \mathrm{eV}$ and $\gamma^{\prime}(20 \mathrm{~K})=20 \mu \mathrm{eV}$ (e.g., see Refs. [20,53]).
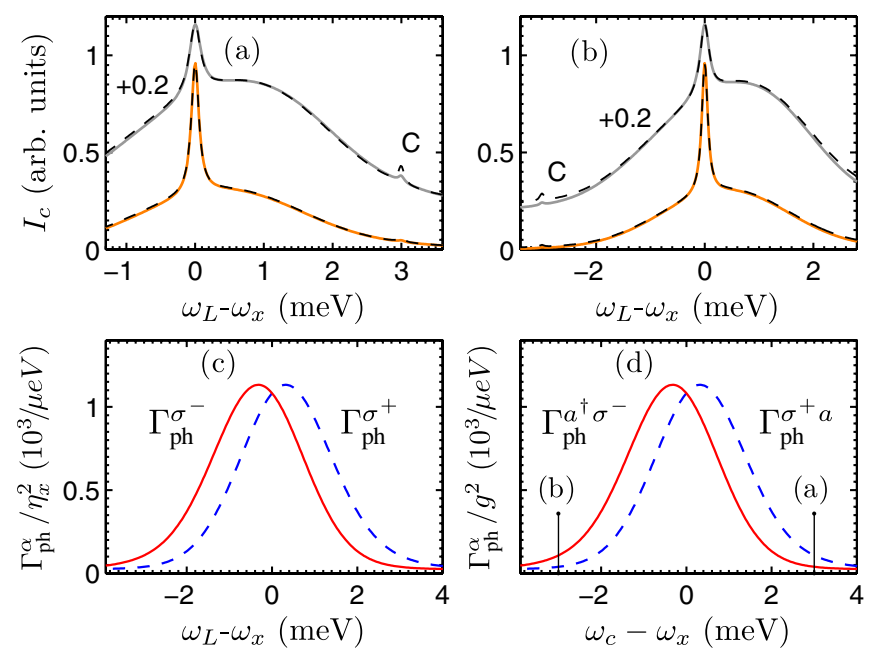

FIG. 6. As in Fig. 5 but with the phonon bath at $T=20 \mathrm{~K}$. The system parameters are identical to those given in Fig. 3, except that $\gamma^{\prime}(20 \mathrm{~K})=20 \mu \mathrm{eV}$ and we now compute $\langle B\rangle(20 \mathrm{~K})=0.73$ $[\mathrm{cf} .\langle B\rangle(4 \mathrm{~K})=0.91]$. For convenience, the scattering rates are plotted in normalized units with respect to $\eta_{x}^{2}$ and $g^{2}$; the two regions marked by the black vertical lines indicate the cavity detunings used in (a) and (b). 
The thermally averaged expectation values of the phonon displacement operators are calculated to be $\langle B\rangle(4 \mathrm{~K})=0.91$ and $\langle B\rangle(20 \mathrm{~K})=0.73$. These results suggest that the dynamics can be well described by our EPME, by essentially only including three separate phonon-scattering effects-since, from the findings above, $L\left(\hat{\sigma}^{+} \hat{a}\right)$ can be safely neglected.

In Fig. 5(c) we plot the phonon-scattering rates, $\Gamma_{\mathrm{ph}}^{\sigma^{-}}$and $\Gamma_{\mathrm{ph}}^{\sigma^{+}}$, as a function of QD-laser detuning for $\eta_{x}=40 \mu \mathrm{eV}$, at $T=4 \mathrm{~K}$; in Fig. 6(c) we plot these scattering rates for $T=20 \mathrm{~K}$. Since the rates depend on QD-laser detuning and the pump strength, they are obviously important for understanding power broadening in a cavity-QED system. In Figs. 5 and 6(d) we plot the rates, $\Gamma^{\sigma^{+} a}$ and $\Gamma^{a^{\dagger} \sigma^{-}}$, as a function of QD-cavity detuning for two different temperatures; the regions marked by the vertical lines indicate the chosen detunings in Figs. 5(a) and 6(a) and 5(b) and 6(b)also note that these particular rates are fixed as a function of QD-laser detuning. As discussed earlier, the scattering term $\Gamma^{\sigma^{+} a}$ describes a process which involves deexciting the QD and exciting the cavity mode, aided through phonon emission or absorption. For convenience, we plot the phonon-scattering rates in normalized units so that the rates can be obtained at any value of $\eta_{x}$ and $g$. For example, considering a phonon-bath temperature of $T=$ $4 \mathrm{~K}$, with a cavity-exciton detuning of $\Delta_{c x}=-1 \mathrm{meV}$, and $g=80 \mu \mathrm{eV}$, then [see Fig. 5(d)] $\Gamma_{\mathrm{ph}}^{a^{\dagger} \sigma^{-}} \approx 0.5 / 10^{3} \times$ $80^{2} \mu \mathrm{eV} \approx 3 \mu \mathrm{eV}$.
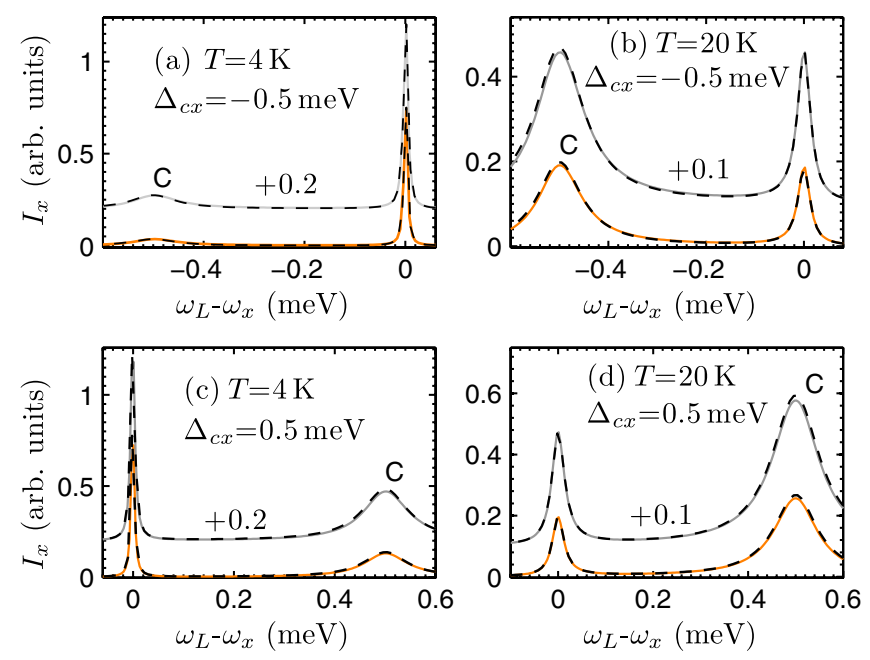

FIG. 7. (a)-(b) Relative QD intensity for a cavity-driven system as a function of QD-laser detuning for $\Delta_{c x}=-0.5 \mathrm{meV}$ and phonon-bath temperatures (a) $T=4 \mathrm{~K}$ and (b) $T=20 \mathrm{~K}$, and for two different values of the $\mathrm{cw}$ laser Rabi frequency (orange solid line corresponds to $\eta_{c}=40 \mu \mathrm{eV}$, and grey, lower, solid line corresponds to $\eta_{c}=60 \mu \mathrm{eV}$ ). Also shown (black dashed curves) is the PL intensity obtained using the effective Lindblad form of the full time-convolutionless ME. The parameters are as follows: $\gamma=2 \mu \mathrm{eV}, \kappa=50 \mu \mathrm{eV}, \gamma^{\prime}=2 \mu \mathrm{eV}$ at $T=4 \mathrm{~K}$, and $\gamma^{\prime}=20 \mu \mathrm{eV}$ at $T=20 \mathrm{~K}$. (c)-(d) Same as in (a) and (b) but with $\Delta_{c x}=0.5 \mathrm{meV}$.
The collective influence from the various phononscattering terms, discussed above, results in the broadening (EID) of the QD exciton resonance, incoherent excitation of the phonon bath, and significant exciton-cavity coupling (or feeding); the first two of these are pump dependent, through $\sim\langle B\rangle^{2} \eta_{x}^{2}$, while the last process scales with $\langle B\rangle^{2} g^{2}$. The trends of the exciton-cavity feeding rate $\Gamma_{\mathrm{ph}}^{a^{\dagger} \sigma^{-}}$are consistent with the results of Refs. [25,26], where one observes a peak scattering rate at around $\Delta_{c x} \sim$ 1-2 meV (depending upon the temperature). Note that at $T=4 \mathrm{~K}$ and $\Delta_{c x}=-3 \mathrm{meV}, \Gamma^{\sigma^{+} a}$ dominates, whereas $\Gamma^{a^{\dagger} \sigma^{-}}$is much larger for $\Delta_{c x}=3 \mathrm{meV}$. However, at $T=20 \mathrm{~K}$, the two rates are much closer to each other for the different detunings as the dependence of the rates on QD-cavity detuning becomes more symmetric with increasing temperature [26].

It is interesting to note that the broadening of the QD exciton resonance as a function of QD-laser detuning closely mirrors the PL line shape associated with the linear exciton spectrum obtained using the IBM. A similar observation was demonstrated by Ahn et al. [69], where the effects of electron-phonon coupling in QDs (with no cavity) on nonstationary resonance fluorescence spectra were studied; the resonance fluorescence dynamics of the QD electronic transition was shown to have a strong dependence on the duration of the laser field, and by increasing the duration of the laser pulse, the background phonon continuum was strongly excited.

TABLE I. Dependence on the $x / c$ FWHM of the intensity PL on $\gamma$, for a QD-driven cavity-measured PL ( $x$ FWHM, with $\Delta_{c x}=3 \mathrm{meV}$ ), and for a cavity-driven QD-measured PL ( $c$ FWHM, with $\Delta_{c x}=0.5 \mathrm{meV}$ ). The QD-cavity parameters are $\kappa=50 \mu \mathrm{eV}, g=20 \mu \mathrm{eV}, \eta_{(x / c)}=30 \mu \mathrm{eV}$, and $\gamma^{\prime}=2 \mu \mathrm{eV}$ ( $4 \mathrm{~K})$. No coupling to phonons is considered and all numbers are in units of $\mu \mathrm{eV}$.

\begin{tabular}{lcc}
\hline \hline & $x$ FWHM (QD-driven) & $c$ FWHM (cavity-driven) \\
\hline$\gamma=2$ & 120 & 107 \\
$\gamma=4$ & 104 & 107 \\
$\gamma=6$ & 98 & 107 \\
\hline \hline
\end{tabular}

TABLE II. Dependence on the $x / c$ FWHM of the powerbroadened line shape on $\kappa$, for a QD-driven cavity-measured system ( $x$ FWHM, with $\Delta_{c x}=3 \mathrm{meV}$ ), and a for cavity-driven QD-measured PL ( $c$ FWHM, with $\Delta_{c x}=0.5 \mathrm{meV}$ ). The QD-cavity parameters are the same as in Table I, and all units are in $\mu \mathrm{eV}$.

\begin{tabular}{lcc}
\hline \hline & $x$ FWHM (QD-driven) & $c$ FWHM (cavity-driven) \\
\hline$\kappa=10$ & 120 & 41 \\
$\kappa=30$ & 120 & 60 \\
$\kappa=50$ & 120 & 107 \\
\hline \hline
\end{tabular}


TABLE III. Dependence on the $x / c$ FWHM of the powerbroadened line shape on $\gamma^{\prime}$, for a QD-driven cavity-measured PL system ( $x$ FWHM, with $\Delta_{c x}=3 \mathrm{meV}$ ), and for a cavitydriven QD-measured PL ( $c$ FWHM, with $\Delta_{c x}=0.5 \mathrm{meV}$ ). The other QD-cavity parameters are the same as in Table I, and all units are in $\mu \mathrm{eV}$.

\begin{tabular}{lcc}
\hline \hline & $x$ FWHM (QD-driven) & $c$ FWHM (cavity-driven) \\
\hline$\gamma^{\prime}=2$ & 120 & 107 \\
$\gamma^{\prime}=4$ & 146 & 107 \\
$\gamma^{\prime}=6$ & 169 & 107 \\
\hline \hline
\end{tabular}

TABLE IV. Dependence on the $x$ FWHM of the powerbroadened PL intensity of a QD-driven cavity-measured system $\left(\Delta_{c x}=3 \mathrm{meV}\right)$, on the two phonon Lindblad processes, $\Gamma_{\mathrm{ph}}^{\sigma^{+}}$and $\Gamma_{\mathrm{ph}}^{\sigma^{-}}$, at $T=4 \mathrm{~K}$, for various values of $\eta_{x}$. The other system parameters are the same as in Table I. Also considered is the case of no incoherent phonon coupling with $\langle B\rangle=1$ (i.e., also no coherent phonon coupling), and $\langle B\rangle=0.91$ at $T=4 \mathrm{~K}$ (with coherent phonon coupling). The inclusion of $\langle B\rangle$ at $4 \mathrm{~K}$ here is to make a better comparison with the same effective $\eta_{x}\left(\rightarrow\langle B\rangle \eta_{x}\right)$ and effective $g(\rightarrow\langle B\rangle g)$; in reality, the no-phonon case will be different because of the lack of coherent phonon renormalizations (later we will show this difference more explicitly, e.g., in Figs. 10-13). All numbers are in units of $\mu \mathrm{eV}$, and note that the broadening here is independent of $\Gamma_{\mathrm{ph}}^{\sigma^{+} a}$ and $\Gamma_{\mathrm{ph}}^{a^{\dagger} \sigma^{-}}$. The last column gives the full polaron ME result [Eq. (11)].

\begin{tabular}{ccccccc}
\hline \hline & $\begin{array}{c}\text { No } \Gamma_{\mathrm{ph}}^{\alpha} \\
\langle B\rangle=1\end{array}$ & $\begin{array}{c}\text { No } \Gamma_{\mathrm{ph}}^{\alpha} \\
\langle B\rangle(4 \mathrm{~K})\end{array}$ & $\Gamma_{\mathrm{ph}}^{\sigma^{+}}$ & $\Gamma_{\mathrm{ph}}^{\sigma^{-}}$ & EPME & Full \\
\hline$\eta_{x}=20$ & 80 & 73 & 71 & 72 & 70 & 72 \\
$\eta_{x}=40$ & 159 & 146 & 138 & 139 & 134 & 151 \\
$\eta_{x}=60$ & 240 & 219 & 251 & 200 & 223 & 390 \\
\hline \hline
\end{tabular}

TABLE V. Dependence on the $c$ FWHM of the powerbroadened PL intensity of a cavity-driven QD-measured system, on the two phonon Lindblad processes, $\Gamma_{\mathrm{ph}}^{\sigma^{+} a}$ and $\Gamma_{\mathrm{ph}}^{a^{\dagger} \sigma^{-}}$, at $T=4 \mathrm{~K}$, for various values of $g$. Also considered is the case of no phonon coupling, with two $\langle B\rangle$ values as above. The general system parameters are the same as in Table I, and all numbers are in units of $\mu \mathrm{eV}$.

\begin{tabular}{lcccc}
\hline \hline & No $\Gamma_{\mathrm{ph}}^{\alpha}\langle B\rangle=1$ & No $\Gamma_{\mathrm{ph}}^{\alpha}\langle B\rangle(4 \mathrm{~K})$ & $\Gamma_{\mathrm{ph}}^{\sigma^{+} a}$ & $\Gamma_{\mathrm{ph}}^{a^{+} \sigma^{-}}$ \\
\hline$g=20$ & 101 & 101 & 101 & 102 \\
$g=40$ & 101 & 101 & 101 & 109 \\
$g=60$ & 101 & 101 & 101 & 115 \\
\hline \hline
\end{tabular}

Similar to the QD-driven system, in Fig. 7 we also investigate the exciton PL characteristics, $I_{x} \propto \bar{n}_{x}$, but with cavity excitation (investigated in more detail in Sec. IV C). As anticipated from Fig. 4, we again obtain a very good fit between our EPME results and the full polaron time-convolutionless ME solution. In general, the effective phonon Lindblad solution is expected to closely
TABLE VI. Dependence on the $c$ FWHM of the powerbroadened PL intensity of a cavity-driven QD-measured system $\left(\Delta_{c x}=0.5 \mathrm{meV}\right)$, on the two Lindblad processes, $\Gamma_{\mathrm{ph}}^{\sigma^{+} a}$ and $\Gamma_{\mathrm{ph}}^{a^{\dagger} \sigma^{-}}$, at $T=4 \mathrm{~K}$, for various values of $\eta_{c}$. Also considered is the case of no phonon coupling with two $\langle B\rangle$ values. The general system parameters are the same as in Table I, and all numbers are in units of $\mu \mathrm{eV}$.

\begin{tabular}{lcccccc}
\hline \hline & $\begin{array}{c}\text { No } \Gamma_{\mathrm{ph}}^{\alpha} \\
\langle B\rangle=1\end{array}$ & $\begin{array}{c}\text { No } \Gamma_{\mathrm{ph}}^{\alpha} \\
\langle B\rangle(4 \mathrm{~K})\end{array}$ & $\Gamma_{\mathrm{ph}}^{a^{\dagger} \sigma^{-}}$ & $\Gamma_{\mathrm{ph}}^{\sigma^{+} a}$ & EPME & Full \\
\hline$\eta_{c}=20$ & 100 & 100 & 100 & 100 & 100 & 100 \\
$\eta_{c}=40$ & 101 & 101 & 101 & 103 & 103 & 103 \\
$\eta_{c}=60$ & 102 & 102 & 102 & 107 & 108 & 108 \\
\hline \hline
\end{tabular}

mimic the full solution here for large QD-cavity detunings $\left(\left|\Delta_{c x}\right| \gg g, \eta_{c}\right)$, though we find that it can work well over a wide range of excitation conditions. Moreover, for cavity excitation, essentially only the $L\left(a^{\dagger} \sigma^{-}\right)$scattering process is needed.

A preliminary analysis of the role of the various scattering processes on the PL intensity is presented in Tables I, II, III, IV, V, and VI. Here we focus on the qualitative influence of the various scattering rates on the exciton $(x)$ and cavity $(c)$ FHWM broadenings, but later we will investigate the full power-broadened PL curves in detail. In Table I we study the dependence of the $x / c$ FWHM of the PL intensity on $\gamma$ (the bare radiative-decay rate). Increasing $\gamma$ results in reduced broadening of a cavitymeasured QD-driven PL line shape ( $x$ FWHM). Increasing $\gamma$ broadens the QD resonance resulting in reduced coupling between the cavity mode and the QD and subsequent narrowing of the cavity-measured QD-driven PL line shape. However, $\gamma$ has little effect on the power broadening of a QD-measured cavity-driven PL intensity ( $c$ FWHM). In Table II we present numerical results for the $x / c$ FWHM of the PL intensity for different values of $\kappa$. Increasing $\kappa$ here has no observable effect on QD-driven cavitymeasured PL intensity, but increasing $\kappa$ results in the increased broadening of a cavity-driven QD-measured PL intensity. In Table III we study the dependence on $\gamma^{\prime}$, i.e., the pure dephasing of the ZPL. Increasing $\gamma^{\prime}$ increases the broadening of QD-driven cavity-measured PL intensity, but has negligible effect on the cavity-driven QD-measured system.

In Table IV we study the role of the two phonon Lindblad processes, $\Gamma_{\mathrm{ph}}^{\sigma^{+}}$and $\Gamma_{\mathrm{ph}}^{\sigma^{-}}$, on a QD-driven cavity-measured system at $T=4 \mathrm{~K}$, for various values of $\eta_{x}$ for our chosen parameters, the other two phonon processes, i.e., $\Gamma_{\mathrm{ph}}^{\sigma^{+} a}$ and $\Gamma_{\mathrm{ph}}^{a^{\dagger} \sigma^{-}}$, do not influence the powerbroadening line shape here. As can be seen, increasing the influence of these effective Lindblad processes by increasing $\eta_{x}$ reduces the linewidth of the cavity-measured PL intensity. We also see that the $x$ FWHM broadening for increasing drives may be drastically underestimated by the effective phonon ME, even though the qualitative trends of 
the full PL intensity are similar; this is because we have neglected the influence of the drive on the phase integrations that enter the full incoherent phonon scattering [see Eqs. (8a) and (11)].

In Table V, we list the $c$ FWHM of the power-broadening line shape of a cavity-driven QD-measured system for the two Lindblad processes, $\Gamma_{\mathrm{ph}}^{\sigma^{+} a}$ and $\Gamma_{\mathrm{ph}}^{a^{\dagger} \sigma^{-}}$, at $T=4 \mathrm{~K}$, for various values of $g$. The Lindblad process caused by $\Gamma_{\mathrm{ph}}^{\sigma^{+} a}$ has no effect on the $c$ FWHM. However, increasing $\Gamma_{\mathrm{ph}}^{a^{\dagger} \sigma^{-}}$ (the cavity feeding process) results in some increased broadening. Finally, in Table VI we show the influence of these phonon processes $\left(\Gamma_{\mathrm{ph}}^{a^{\dagger} \sigma^{-} / \sigma^{+} a}\right)$ with increasing $\eta_{c}$, and these calculated rates also show a slight increase of the $c$ FWHM with increasing drives. Now we see that the $c$ FWHM broadening for increasing drives is extremely well reproduced with the effective phonon ME; this is because the cavity pump no longer enters $H_{\text {sys }}^{\prime}$ [see Eqs. (9a) and (11)]. Note, in both Tables V and VI, we see no role from the slight coherent reduction of $\langle B\rangle g$ at $4 \mathrm{~K}$.

To summarize this subsection, we have identified three main Lindblad phonon processes that contribute to the PL intensity of a cavity-QED system. We have also found earlier that the effective scattering rates associated with these processes can be calculated, under certain detuning conditions, from simple analytical solutions [Eqs. (18) and (19)]; these effective Lindblad solutions are compared to the full polaron ME solution and found, in certain cases, to yield very good agreement. In this way we can also argue the underlying physics of the identified phonon-scattering processes. However, there can be noticeable differences, especially for the measured $x$ FWHM broadening of an exciton-driven system. In general, we also recognize that driving via the exciton or the cavity can yield drastically different results.

In what follows below, we will use the full polaron ME, and first verify the general need for multiphonon and mutiphoton effects.

\section{B. Influence of multiphonons and multiphotons on the PL intensity}

Our time-convolutionless ME above [Eq. (11)] utilizes the polaron frame, which allows for a nonperturbative treatment of phonons. This enables one to use the full IBM machinery to compute the phonon correlation functions. It is also useful to look at the one-phonon limit of the time-convolutionless ME by expanding the phonon Green functions to lowest order in the phonon coupling. In this limit we can expand the phonon correlation function $\phi(t)$ to lowest order in the dot-phonon-coupling constant as follows: $G_{g}(t) \simeq 0$ and $G_{u}(t) \simeq \phi(t)$, where we have used $\langle B\rangle \simeq 1$. This then connects to a weak-phonon coupling (i.e., perturbative) approach.

In Figs. 8(a) and 8(b) we plot the cavity-mode intensity $\left(I_{c}\right)$ for an exciton-driven system as a function of
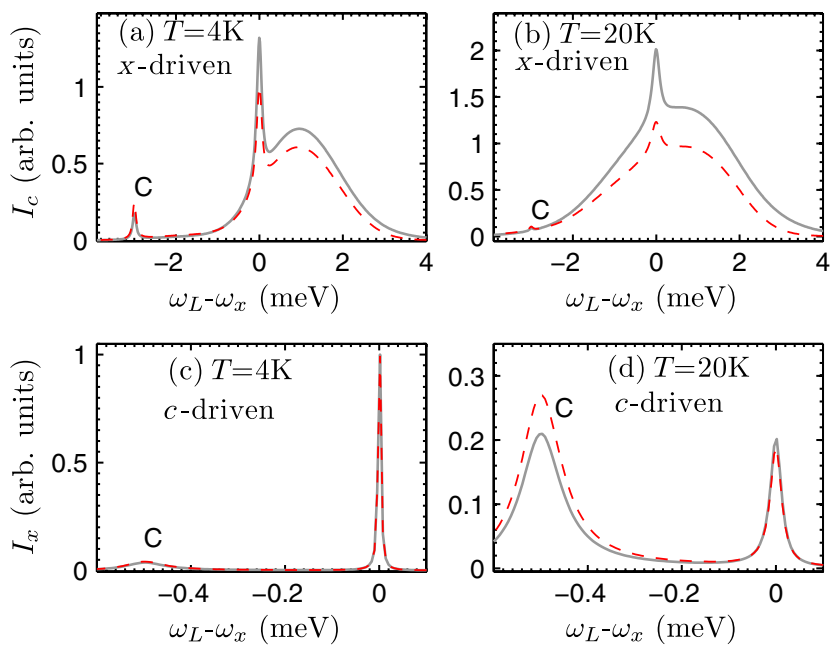

FIG. 8. (a)-(b) Cavity-mode PL intensity for a QD-driven system $\left(\eta_{x}=30 \mu \mathrm{eV}\right)$ as a function of QD-laser detuning for a onephonon theory (red dashed line) and the full polaron model (grey solid line) with $\Delta_{c x}=-3 \mathrm{meV}$, for phonon-bath temperatures (a) $T=4 \mathrm{~K}$ and (b) $T=20 \mathrm{~K}$. (c)-(d) Relative QD (exciton) intensity for a cavity-driven system $\left(\eta_{c}=30 \mu \mathrm{eV}\right)$ as a function of QD-laser detuning for a one-phonon theory (red dashed line) and the full polaron model (grey solid line) with $\Delta_{c x}=-0.5 \mathrm{meV}$, for phonon-bath temperatures (c) $T=4 \mathrm{~K}$ and (d) $T=20 \mathrm{~K}$.

exciton-laser detuning for a one-phonon solution and the full polaron (multiphonon) solution. We recognize that onephonon-scattering process tends to deviate from the full polaron PL intensity, which is especially noticeable at higher temperatures and for larger driving-field strengths. Thus, even for low temperatures, a weak phonon-coupling theory can break down. We further find that the one-phonon approximation overestimates the power-broadening PL intensity for a negatively detuned cavity. Similar conclusions about the need for multiphonon effects were also found in the context of the resonance fluorescence spectra of a QD-driven cavity-QED system [40] and time-dependent excitonic Rabi rotation dynamics [41]. However, for a cavity-driven system [Fig. 8(c) and 8(d)], at low temperatures (e.g., $4 \mathrm{~K}$ ), the weak-phonon theory can be accurate. Of course, other QD material parameters can yield different trends in the role and assessment of multiphonon coupling. Since the phonon correlation functions that we use are well known for an IBM model, the full phonon calculation of the PL intensity presents the same level of computational complexity as the one-phonon calculations, which is a major advantage of the ME formalism above. Even at the Lindblad $\mathrm{ME}$ level, it is just as easy to use a full polaron solution.

We also study the influence of quantized multiphoton processes in the PL intensity of both an exciton-driven and a cavity-driven system. In Figs. 9(a) and 9(b), using cavity excitation $\left(\eta_{c}=30 \mu \mathrm{eV}\right)$, we plot $I_{x}$ as a function of QD-laser detuning for various truncations of the photon Hilbert state. We find that it is necessary to include up to six-photon processes (e.g., a 13 state model) to correctly 

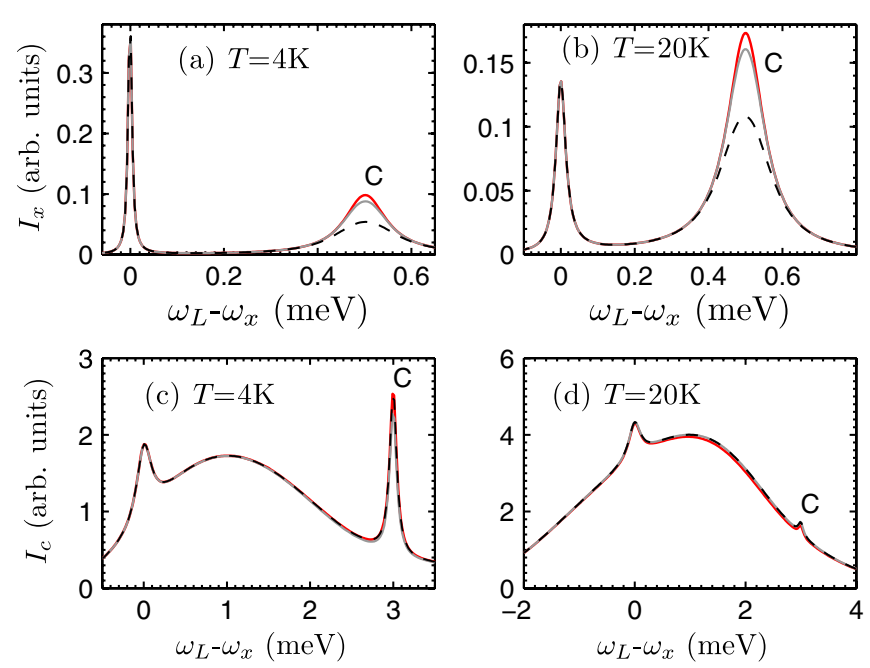

FIG. 9. (a)-(b) Exciton PL intensity for a cavity-driven system $\left(\eta_{c}=30 \mu \mathrm{eV}\right)$ as a function of QD-laser detuning (with $\Delta_{c x}=$ $0.5 \mathrm{meV}$ ), for different numbers of truncated photon states at (a) $T=4 \mathrm{~K}$ and (b) $T=20 \mathrm{~K}$ : two-photon (or more, since this value has converged): black dashed line, four-photon: grey solid line, six-photon (or more): red solid line. (c)-(d) Mean cavityphoton number for a dot-driven system as a function of QD-laser detuning (with $\Delta_{c x}=3 \mathrm{meV}$ ), for a different number of truncated photon states with (c) $T=4 \mathrm{~K}$ and (d) $T=20 \mathrm{~K}$ : one-photon: black dashed line, two-photon: grey solid line, and three-photon: red darker line.

describe the effect of cavity photons; note that including more than six-photon processes yields an identical result to the six-photon calculations, so these values have converged on the numerically exact answer. While the need for six-photon processes may seem surprising for the relatively small values of $g$ (i.e., $20 \mu \mathrm{eV}$ ), the exciton-phonon coupling with phonon scattering can be more sensitive to cavity-QED effects-especially with coherent excitation. In the rest of the paper, we thus compute the PL intensity of the cavity-driven system in a six-photon truncated basis.

In Figs. 9(c) and 9(d) we plot the cavity PL intensity $\left(I_{c}\right)$ for a QD-driven system $\left(\eta_{x}=30 \mu \mathrm{eV}\right)$, as a function of exciton-laser detuning for various truncations of the photon Hilbert state. Unlike a cavity-driven system, we now find that a two-photon truncation of the photon Hilbert space is enough to obtain a correct (i.e., converged in the photon basis) description of the cavity PL. The fundamental difference between a QD-driven and a cavity-driven system is due to the fact that the cavity is represented as a quantized harmonic oscillator whereas a QD is a two-level system. Hence, for our system parameters, the cavity is more easily excited into higher lying Jaynes-Cummings ladder states, even though the system exhibits relative strong dissipation (i.e., $\kappa=2.5 \mathrm{~g}$ ). Neglecting quantum multiphoton processes can therefore introduce spurious effects in the PL line shape. A two-photon truncation was also found to be sufficient (and necessary) for the study of the fluorescence spectrum (Mollow triplet) of a QD-driven cavity-QED system [40].
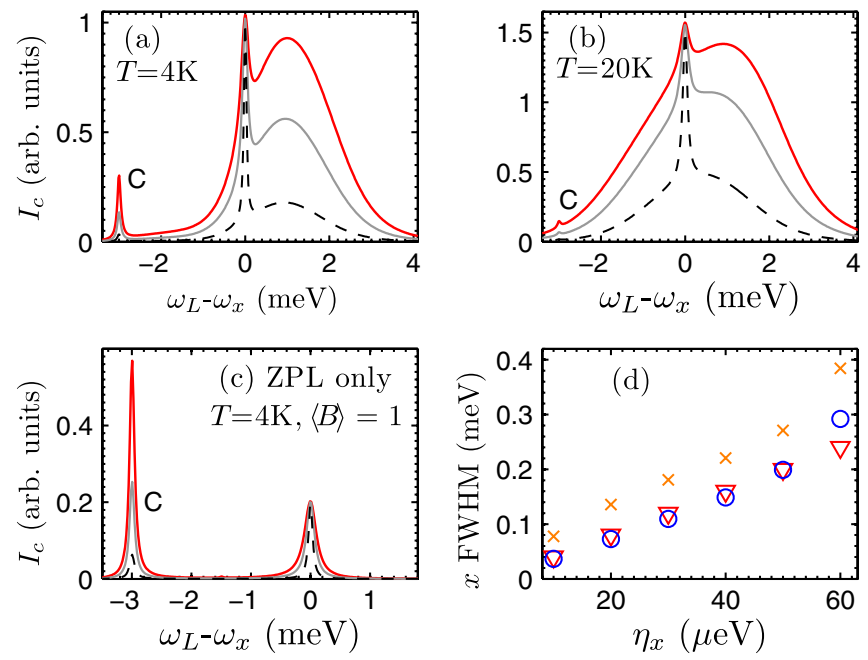

FIG. 10. (a)-(b) Relative cavity-mode intensity as a function of QD-laser detuning for a dot-driven system at two different temperatures, (a) $T=4 \mathrm{~K}$ and (b) $T=20 \mathrm{~K}$, and for different values of the cw Rabi frequency $\left(\eta_{x}=20 \mu \mathrm{eV}\right.$ : black dashed line, $\eta_{x}=40 \mu \mathrm{eV}$ : grey solid line, and $\eta_{x}=60 \mu \mathrm{eV}$ : red solid line). The $\mathrm{cw}$ field drives the QD which is detuned to the right of the cavity mode by $3 \mathrm{meV}\left(\Delta_{c x}=-3 \mathrm{meV}\right)$. Note that $\langle B\rangle=$ 0.91 at $T=4 \mathrm{~K}$ and $\langle B\rangle=0.73$ at $T=20 \mathrm{~K}$. (c) Normalized cavity-mode intensity in the presence of only ZPL broadening with $\langle B\rangle=1$ at $T=4 \mathrm{~K}$. (d) Plot of the $x$ FWHM of the PL intensity at the QD resonance as a function of $\eta_{c}$. The orange crosses show the FWHM at $T=20 \mathrm{~K}$, the blue circles at $T=$ $4 \mathrm{~K}$, and the red inverted triangles show the intensity FWHM with only ZPL broadening and $\langle B\rangle=1$ (no phonons).

In summary, the results above highlight the need for both multiphonon and multiphoton effects for understanding power-broadened PL intensity, even for low temperatures $(4 \mathrm{~K})$ and rather small cavity-dot coupling rates $(g=20 \mu \mathrm{eV}$, cf. $\kappa=50 \mu \mathrm{eV})$.

\section{Power broadening through coherent exciton pumping and cavity emission}

Next we study the cavity-emitted PL for different input powers of an exciton pump. In Figs. 10(a) and 10(b) we plot the relative cavity-mode intensity $\left(I_{c}\right)$ as a function of exciton-laser detuning for a QD-driven system at two temperatures, (a) $T=4 \mathrm{~K}$ and (b) $T=20 \mathrm{~K}$, for various values of $\eta_{x}$. The cw field drives the QD which is now detuned to the right (higher energy) of the cavity mode by $3 \mathrm{meV}$. In Fig. 10(c) we plot the relative cavity-mode intensity with only ZPL broadening and set $\langle B\rangle=1$ (i.e., no coherent or incoherent coupling effects from phonons). This then closely corresponds to an atomiclike powerbroadening model, but with the addition of the pure dephasing of the ZPL-a model that is commonly used to analyze semiconductor cavity-QED experiments. The power-broadened intensity line shape in the absence of phonon coupling can be explained by considering two Lorentzian line shapes centered at the two resonances, 
the relative oscillator strengths of which are qualitatively determined by their corresponding broadenings.

Without phonon coupling, as shown in Fig. 10(c), an increasing $\mathrm{cw}$ drive causes power broadening of the QD exciton, which decreases the oscillator strength relative to the cavity mode and also excites the cavity resonance more. With phonon-scattering processes [see Fig. 10(a)], the first major difference we notice is the apparent narrowing of the QD resonance compared to Fig. 10(c), which is due to the coherent renormalization of the Rabi frequency-this manifests in an effective drive whose magnitude decreases with increasing temperature; in addition, the phonon interactions reduce the dot-cavity coupling through $g \rightarrow\langle B\rangle g$. Incoherent phonon coupling also introduces significant additional broadening of the QD exciton due to the $L\left(\sigma^{+}\right)$ process (see Table I), eventually resulting in a new peak near the phonon cutoff frequency (i.e., at the peak of the spectral bath function for phonons, $\omega_{b}=1 \mathrm{meV}$ ). The mean cavity-photon numbers (and thus $I_{c}$ ) increase in the presence of phonons due to phonon-assisted processes whose magnitude also increases with temperature [see Fig. 10(c), which also has a larger ZPL). Comparing the cavity PL characteristics in Figs. 10(a)-10(c), we see that electron-phonon coupling plays a significant role in determining the PL intensity, with features that are not at all explained with simple atomiclike MEs.

In Fig. 10(d) we plot the $x$ FWHM of $I_{c}$ at the QD resonance as a function of $\eta_{x}$, which is a typical measurement in experimental studies [35]. The orange crosses show the intensity at $T=20 \mathrm{~K}$, the blue circles at $T=4 \mathrm{~K}$, and the inverted red triangles show the FWHM in the absence of any phonon coupling. In spite of a significant reduction of the effective Rabi frequency due to phonon coupling at high temperatures (e.g., $\langle B(4 \mathrm{~K})\rangle=0.91 \rightarrow\langle B(20 \mathrm{~K})\rangle=0.77$ ), the $x$ FWHM at $T=20 \mathrm{~K}$ is higher than the FWHM calculated at $T=4 \mathrm{~K}$, which suggests that EID more than compensates for phonon-induced renormalization of the Rabi
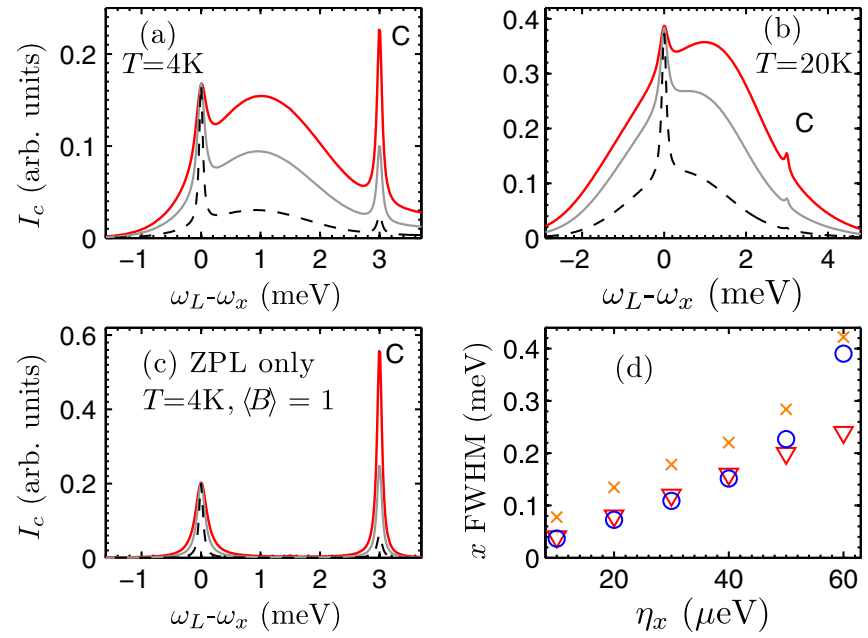

FIG. 11. As in Fig. 10 [cf. $\Delta_{c x}=-3 \mathrm{meV}$ in Fig. 10]. frequency. It was earlier noted in Table I that increasing $\gamma$ (and thus also $\Gamma^{\sigma^{-}}$, as they have the same Lindblad operators) reduces the mean cavity-photon number. We also note from Table IV that the primary contribution to the broadening comes from the two scattering terms $\Gamma_{\mathrm{ph}}^{\sigma^{+}}$and $\Gamma_{\mathrm{ph}}^{\sigma^{-}}$; these two processes increase with temperature and driving strength and introduce additional broadening, which reduces $I_{c}$. At a pump rate of $\eta_{x} \sim 60 \mu \mathrm{eV}$, we see a more rapid increase of the $x$ FWHM with phonon coupling due to stronger $\mathrm{cw}$ laser-phonon coupling. For pump rates greater than $\eta_{x} \sim 40 \mu \mathrm{eV}$, we also see a more rapid increase of the $x$ FWHM with phonon coupling.

In Figs. 11(a) and 11(b) we plot the relative cavity-mode PL intensity for a dot-driven system, where the exciton resonance is now detuned to the left of the cavity mode by $3 \mathrm{meV}$. We again consider two different temperatures of the phonon reservoir, (a) $T=4 \mathrm{~K}$ and (b) $T=20 \mathrm{~K}$, for various $\eta_{x}$. In Fig. 11(c) we show the normalized cavity-mode intensity with only ZPL broadening and $\langle B\rangle=1$. The generic features of Fig. 11 can be understood exactly along the lines of the arguments presented above for Fig. 10. However, as discussed earlier, here we obtain a significant reduction in the mean cavity-photon number (less cavity feeding) since the cavity is now energetically higher, which requires absorption of phonons-compare Figs. 10(a) and 11(a). Moreover, the mean cavity-photon number is smaller than that in the absence of phonons due to the renormalized (reduced) dot-cavity coupling which, however, increases with temperature. In Fig. 11(d) we plot the $x$ FWHM of the cavity emission as a function of $\eta_{x}$ which shows very similar features to the $x$ FWHM data presented in Fig. 10(d). We remark that the approximate $x$ FWHM values are extracted numerically by fitting with a simple Lorentzian model. As it is clear from the plots, the power-broadening PL intensity in the presence of phonons is no longer represented by a simple system of coupled Lorentzians, and we observe pronounced non-Lorentzian line shapes and clear signatures from the phonon-bath spectral function. We reiterate the point that there is a substantial discrepancy at large pumping fields between the $x$ FWHM from an effective Lindblad solution (EPME) and a full polaron ME solution (see Table IV). This highlights a breakdown of the EPME at large pumping fields, which is not too surprising given the rather coarse approximations made in its derivation. Finally, we comment that the $c$ FWHM (which can be extracted from the same PL data) does not show any power broadening, which is possibly also a result of the reasonably large QD-cavity detunings.

\section{Power broadening through coherent cavity pumping and exciton emission}

In this subsection, we focus on a cavity-excited system, where the emitted excited intensity $\left(I_{x}\right)$ is detected through the QD emission, e.g., through (non-cavity mode) radiation 

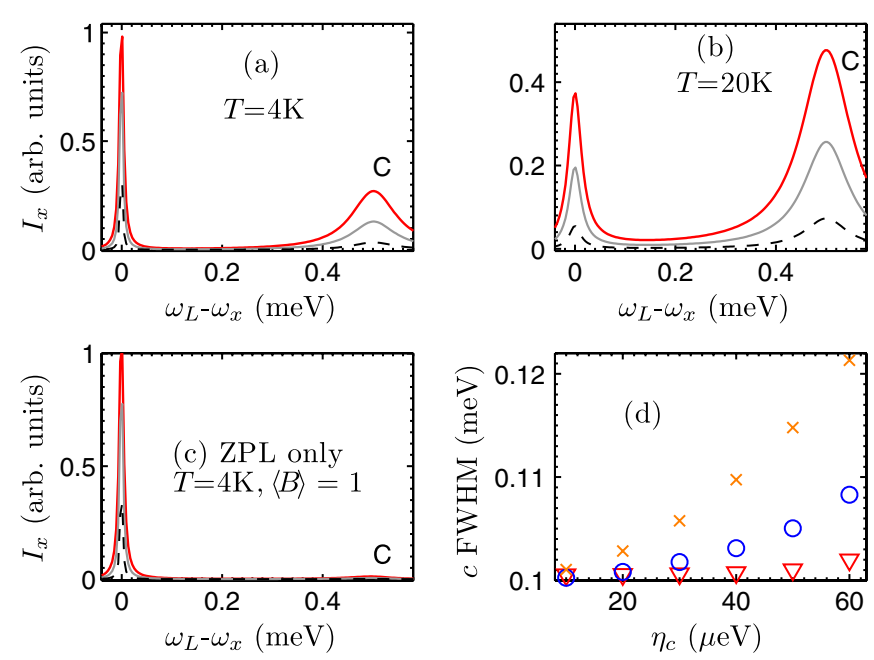

FIG. 12. As in Fig. 10, but for the exciton intensity obtained in the presence of a cavity pump. The $\mathrm{cw}$ drive strengths are $\eta_{c}=$ 10, 40, $60 \mu \mathrm{eV}$. In (d), the orange crosses show the $c$ FWHM at $T=20 \mathrm{~K}$, the blue circles at $T=4 \mathrm{~K}$, and the inverted red triangles show the FWHM with only ZPL broadening and $\langle B\rangle=$ 1. The cavity-exciton detuning is $\Delta_{c x}=0.5 \mathrm{meV}$.

modes. In Figs. 12(a) and 12(b) we plot the relative exciton intensity $I_{x}$ as a function of QD-laser detuning for a cavitydriven system at two different temperatures, (a) $T=4 \mathrm{~K}$ and (b) $T=20 \mathrm{~K}$, for various values of $\eta_{c}$. The cw field drives the cavity mode, which is now detuned to the right of the QD exciton by $0.5 \mathrm{meV}$. In Fig. 12(c) we show $I_{x}$ with no phonon interactions and $\langle B\rangle=1$ (i.e., no coherent or incoherent effects from phonons, apart from pure dephasing). We discern that the power-broadened PL intensity in the absence of phonons is quite distinct from the case with finite phonon coupling. On the one hand, we have lost the resonance at the phonon spectral function since there is no longer a term that involved incoherent excitation through the phonon reservoir $\left(\Gamma_{\mathrm{ph}}^{\sigma^{+}}\right.$process). On the other hand, we see a clear influence from phonon-induced exciton-cavity feeding. In particular, without phonon coupling we observe very little emission at the cavity-mode frequency, which further demonstrates that phonons play a significant role in the power-broadened PL line shape through dot-cavity coupling (via cavity feeding). Also note that the broadening of the QD resonance increases due to enhanceddot-cavity coupling in the presence of phonons, which increases with temperature. However, at higher temperatures the mean exciton number is decreased, which is mainly due to phonon-induced reduction in $g \rightarrow\langle B\rangle g$.

In Fig. 12(d) we plot the $c$ FWHM obtained via exciton emission $\left(I_{x}\right)$, as a function of $\eta_{c}$. The $c$ FWHM in the presence of phonons at low temperatures $(T=4 \mathrm{~K})$ is very similar to the case with only ZPL broadening, primarily because of the absence of EID, as the system is now cavity driven, though $g \rightarrow\langle B\rangle g$ is still temperature dependent.
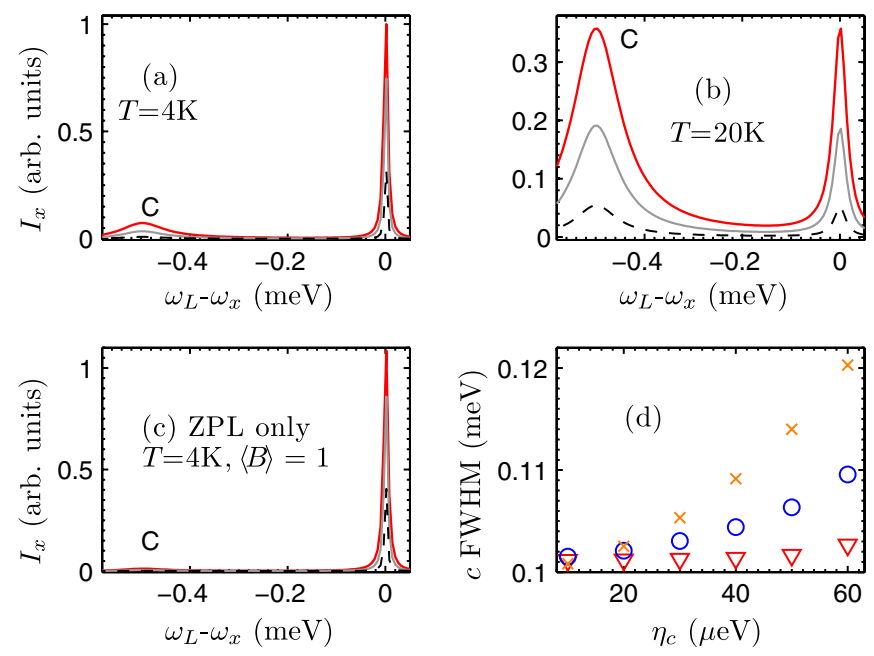

FIG. 13. As in Fig. 12, but for $\Delta_{c x}=-0.5 \mathrm{meV}$.

However, the $c$ FWHM increases with temperature even though $\langle B\rangle g$ decreases. We also note that the emission at the QD frequency is suppressed with increasing temperatures. With further increase in temperatures (i.e., above $20 \mathrm{~K}$ - not shown), the PL intensity is dominated by emissions at the cavity mode. Similar to the exciton pumped system, for pump rates of $\eta_{c} \sim 40 \mu \mathrm{eV}$, we observe a more rapid increase of the $c$ FWHM with phonon coupling. For cavity excitation, the effective Lindblad solution shown earlier provides a very good match for the $c$ FWHM for all $\eta_{c}$ pump values studied (see Table VI). This is primarily because the approximations made for a cavity-driven system are less restrictive. For instance, there are only two Lindblad terms (and only one of these is important here), which is unlike the case of a QD-driven system where four Lindblad terms are needed (two of which are $\eta_{x}$ dependent).

In Figs. 13(a) and 13(b) we again plot the relative exciton intensity $\left(I_{x}\right)$ as a function of QD-laser detuning for a cavity-driven system, but now with the cavity-mode detuned to the left (lower energy) of the QD exciton by $0.5 \mathrm{meV}$. In Fig. 13(c) we show the normalized cavity-mode intensity with only ZPL broadening of the exciton and $\langle B\rangle=1$. We highlight the significant difference in the QD emission at the cavity-mode energy for the two detunings at low temperature [Fig. 12(a) and 13(a)] - the cavity-mode resonance is more pronounced with a positive $\Delta_{c x}$. This difference is due to the asymmetry in the phonon absorption and emission probability; at higher temperatures, however, the line shapes becomes more similar. In Fig. 13(d) we plot the $c$ FWHM obtained through exciton emission as a function of the cw laser Rabi frequency $\left(\eta_{c}\right)$, which is very similar to that in Fig. 12(d). For these studies, the $c$ FWHM is mostly independent of the detuning of the dot-cavity system. We also mention that $x$ FWHM (which can be extracted from the same PL data) only shows marginal power broadening, though it may increase with reduced QD-cavity detuning. 

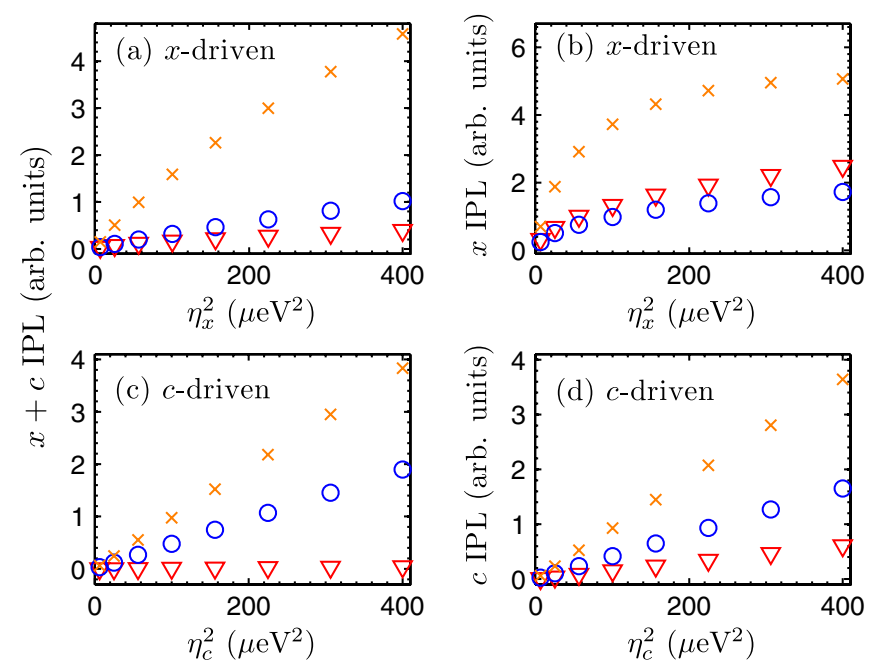

FIG. 14. Integrated PL for a dot-driven system, (a) and (b), as measured through cavity emission, and the IPL of a cavitydriven system, (c) and (d), as measured via QD emission; both as a function of the square of the $\mathrm{cw}$ drive for two different temperatures of the phonon reservoir $(T=4 \mathrm{~K}$ : blue circles, $T=20 \mathrm{~K}$ : orange crosses); also plotted are the IPL line shape in the presence of only ZPL broadening and $\langle B\rangle=1$ (inverted red triangles) - the corresponding $\gamma^{\prime}=2 \mu \mathrm{eV}$ (at $4 \mathrm{~K}$ ). (a) The IPL of the total cavity and QD intensity of a dot-driven system as measured through cavity emission; $\Delta_{c x}=3 \mathrm{meV}$ and the PL intensity are shown in Fig. 11. (b) IPL of the cavity-mode intensity over the Lorentzian centered at the QD resonance frequency. (c) IPL of the QD exciton and cavity intensity of a cavity-driven system as measured through the QD; here $\Delta_{c x}=$ $0.5 \mathrm{meV}$ and the intensity line shapes are shown in Fig. 13. (d) The IPL of the QD mode intensity over the Lorentzian centered at the cavity resonance frequency.

\section{E. Integrated photoluminescence}

Finally, we study the IPL. In Figs. 14(a) and 14(b) we plot the IPL line shape for a dot-driven system as measured through the cavity, for the positively detuned cavity mode $\left(\Delta_{c x}=3 \mathrm{meV}\right)$. The total IPL of the cavity and exciton intensities of a dot-driven system plotted in Fig. 14(a) does not saturate with increasing drives-unlike the IPL over the Lorentzian centered at the exciton resonance frequency plotted in Fig. 14(b) (i.e., the integrated $x$ FWHM, emitted via the cavity mode), which shows clear saturation. The Lorentzian line shape at the QD exciton resonance is computed by subtracting off the background phonon sidebands from the PL intensity. The lack of saturation of the IPL of the total cavity and QD intensity is attributed to the increasing excitation of the background phonon continuum with increasing $\mathrm{cw}$ drives, which is also enhanced at higher temperatures. These theoretical trends are consistent with the experimental results of Ates et al. [35].

In Figs. 14(c) and 14(d) we show the IPL of the cavitydriven system (with $\Delta_{c x}=0.5 \mathrm{meV}$ ) as measured via the QD exciton emission. For our chosen parameters, neither the total IPL of the cavity and exciton intensity of a cavity-driven system plotted in Fig. 14(c), nor the IPL over the Lorentzian centered only at the cavity resonance frequency plotted in Fig. 14(d) (i.e., the integrated $c$ FWHM, emitted via the QD) show any saturation. Furthermore, significant power broadening is found, which increases with the temperature of the phonon bath because of enhanced phonon-mediated coupling between the dot and the cavity mode.

\section{CONCLUSIONS}

We have presented a detailed analysis of the PL intensity power broadening of a semiconductor cavity-QED system under the coherent excitation of either the QD exciton or the cavity mode. In particular, we have included the interaction of the QD with an acoustic-phonon environment at a microscopic level, while also accounting for exciton-cavity coupling in the regime of cavity-QED. We utilized a timeconvolutionless ME approach in the polaron frame to study the cavity-QED dynamics. The interaction of the phonon reservoir with the QD is nonperturbative and is limited mainly by the validity of the IBM (and the single exciton picture) at high temperatures. This approach enables us to treat the coherent interaction between the QD, cavity, and the cw laser field to all orders. Central to this approach is the need to account for the internal coupling effects, which preserves the detail balanced condition on the system density operator. Our theory also points out some major flaws and restrictions of atomiclike MEs for modeling coherent excitation regimes in semiconductor QD-cavity systems.

Using material parameters close to those measured in related semiconductor experiments, various PL intensity line shapes were studied as a function of the excitation pump rate, for different temperatures of the phonon bath. We computed the full PL intensity line shape over a range of frequencies and extracted approximate Lorentzianlike linewidths of the $c / x$ FWHM of the power-broadening PL intensity for both the QD-driven and cavity-driven systems. The interaction of the QD with the phonon reservoir is seen to introduce qualitatively different features in the PL intensity, especially at higher temperatures, and is quite distinct from the case of a typical power-broadening line shape of a two-level atom. We found a significant narrowing of the QD resonance due to the coherent renormalization of the Rabi frequency resulting in a reduced effective drive. The interaction with the phonon reservoir also reduced the dot-cavity coupling due to the renormalization of $g \rightarrow\langle B\rangle g$. Phonon coupling further introduces additional broadening of the QD exciton due to EID and results in highly nonLorentzian line shapes for the PL intensity; by way of incoherent excitation from the phonon bath, clear signatures of the phonon-bath spectral function appear in the exciton-driven PL. For a cavity-driven system, the mean cavity-photon number (which is proportional to the cavitymode intensity) was also found to change in the presence of 
phonon-assisted processes, which depended sensitively on dot-cavity detuning and the temperature of the phonon bath.

To help explain the underlying physics of electronphonon scattering in these cavity-QED systems, we derived an effective phonon ME (EPME) of the Lindblad form, which facilitates a very simple numerical solution to the full ME and also allows the extraction of various phonon-induced scattering rates in a physically meaningfully way. In particular, we identified specific phononmediated processes which cause the EID of the QD exciton resonance and incoherent exciton pumping. We identified Lindblad superoperators that mediate incoherent interactions between the QD and the cavity mode, resulting in exciton-cavity feeding - a phenomenon that is becoming more familiar in semiconductor cavity-QED (e.g., see Refs. [13,18,20-22,24-27,29,40]). We also studied the regimes of validity of the effective Lindblad solution and found that, for relatively large QD-cavity detunings, the effective Lindblad solution produces a very good fit to the full polaron ME solution. However, discrepancies can occur for exciton pumped systems with increasing drives, where one requires the full polaron ME. Using these polaronic ME formalisms, we found that phonon-induced EID and incoherent coupling between the QD and the cavity is fundamental to obtaining a complete picture of power broadening in these semiconductor systems. In particular, our results demonstrate that the cavity-emitted QD-driven PL intensity can display quite profound signatures of the phonon-bath spectral function. A cavity-driven system also contains clear signatures of the phonon bath, containing power-broadened features that are significantly different from those obtained in atomic QED. We have also demonstrated that the pure dephasing of the ZPL, frequently cited as being responsible for phonon-scattering effects such as cavity feeding, actually only plays a very minor role here; the physics of the phonon-scattering processes that we identity are manifestly different to the physics of coupled Lorentzian oscillators, either with or without the pure dephasing of the ZPL.

The results presented in this paper should be of broad interest to those interpreting current and future experimental data from semiconductor cavity-QED systems. A more rigorous analysis would use the full polaron $\mathrm{ME}$ and a less rigorous approach would use the EPME; the latter approach allows for a simple and intuitive picture of the underlying physics and can be accurate in certain excitation regimes. Our power-broadening results were chosen to highlight new regimes where phonon effects are more readily visible and can serve to direct experimental focus in those regimes. For this work, we have also focused our studies to the domain of cw excitation, but the extension to pulsed systems is straightforward and will be presented elsewhere.

\section{ACKNOWLEDGMENTS}

This work was supported by the National Sciences and Engineering Research Council of Canada and the Canadian Foundation for Innovation. We thank H. J. Carmichael for useful discussions.

\section{APPENDIX: EFFECTIVE LINDBLAD MASTER EQUATIONS WITH SIMPLIFIED ELECTRON- PHONON COUPLING}

In this Appendix we provide some technical details into the derivation of our effective Lindblad master equation, Eq. (15). We exemplify the relatively simple case of a cavity-driven system, where $\hat{X}_{g}=\hbar g\left(\hat{a}^{\dagger} \hat{\sigma}^{-}+\hat{\sigma}^{+} \hat{a}\right)$ and $\hat{X}_{u}=i \hbar g\left(\hat{\sigma}^{+} \hat{a}-\hat{a}^{\dagger} \hat{\sigma}^{-}\right)$. The case of a dot-driven system can also be derived using similar steps but with a few additional approximations discussed at the end of this Appendix. The integrand inside the phonon integral in Eq. (11), $\int_{0}^{\infty} d \tau \sum_{m=g, u}\left\{G_{m}(\tau)\left[\hat{X}_{m}, e^{-i H_{\mathrm{sys}}^{\prime} \tau / \hbar} \hat{X}_{m} \times\right.\right.$ $\left.e^{i H_{\mathrm{sys}}^{\prime} \tau / \hbar} \rho(t)\right]+$ H.c.\}, can be approximated (e.g., for $m=g$ ) as follows:

$$
\begin{aligned}
& G_{g}(\tau)\left[\hat{X}_{g}, e^{-i H_{\mathrm{sy}}^{\prime} \tau / \hbar} \hat{X}_{g} e^{i H_{\mathrm{sy}}^{\prime} \tau / \hbar} \rho(t)\right]+\text { H.c. } \\
& \simeq \hbar^{2} g^{2} G_{g}(\tau)\left(\hat{a}^{\dagger} \hat{\sigma}^{-}+\hat{\sigma}^{+} \hat{a}\right)\left(\hat{a}^{\dagger} \hat{\sigma}^{-} e^{-i \Delta_{c x} t}+\hat{\sigma}^{+} \hat{a} e^{i \Delta_{c x} t}\right) \rho(t)-\hbar^{2} g^{2} G_{g}(\tau)\left(\hat{a}^{\dagger} \hat{\sigma}^{-} e^{-i \Delta_{c x} t}+\hat{\sigma}^{+} \hat{a} e^{i \Delta_{c x} t}\right) \rho(t)\left(\hat{a}^{\dagger} \hat{\sigma}^{-}+\hat{\sigma}^{+} \hat{a}\right) \\
& +\hbar^{2} g^{2} G_{g}^{*}(\tau) \rho(t)\left(\hat{a}^{\dagger} \hat{\sigma}^{-} e^{-i \Delta_{c x} t}+\hat{\sigma}^{+} \hat{a} e^{i \Delta_{c x} t}\right)\left(\hat{a}^{\dagger} \hat{\sigma}^{-}+\hat{\sigma}^{+} \hat{a}\right)-\hbar^{2} g^{2} G_{g}^{*}(\tau)\left(\hat{a}^{\dagger} \hat{\sigma}^{-}+\hat{\sigma}^{+} \hat{a}\right) \rho(t)\left(\hat{a}^{\dagger} \hat{\sigma}^{-} e^{-i \Delta_{c x} t}+\hat{\sigma}^{+} \hat{a} e^{i \Delta_{c x} t}\right),
\end{aligned}
$$

where we have used

$$
e^{-i H_{\mathrm{sys}}^{\prime} \tau / \hbar} \hat{X}_{m} e^{i H_{\mathrm{sys}}^{\prime} \tau / \hbar} \simeq e^{-i H_{0}^{\prime} \tau / \hbar} \hat{X}_{m} e^{i H_{0}^{\prime} \tau / \hbar},
$$

with $H_{0}^{\prime}=\hbar \Delta_{x L} \hat{\sigma}^{+} \hat{\sigma}^{-}+\hbar \Delta_{c L} \hat{a}^{\dagger} \hat{a}$. This corresponds to approximating $H_{\text {sys }}^{\prime}$ with $H_{0}^{\prime}$ in the exponential phase and is expected to be valid only when the dot-cavity detuning is large compared to $g$. It follows that

$$
\begin{gathered}
e^{-i H_{0}^{\prime} \tau / \hbar} \hat{a}^{\dagger} \hat{\sigma}^{-} e^{i H_{0}^{\prime} \tau / \hbar}=e^{-i \Delta_{c x} t} \hat{a}^{\dagger} \hat{\sigma}^{-}, \\
e^{-i H_{0}^{\prime} \tau / \hbar} \hat{\sigma}^{+} \hat{a} e^{i H_{0}^{\prime} \tau / \hbar}=e^{i \Delta_{c x} t} \hat{\sigma}^{+} \hat{a} .
\end{gathered}
$$

Consequently, this allows us to write an effective Lindblad ME that can (e.g., see Figs. 3-5) reproduce the full polaron ME solution over a range of dot-laser and cavity-exciton detunings. The major advantage of this approach (used on its own or as a compliment) is that it is significantly simpler than the full polaron ME approach and allows one to extract various, physically meaningful, scattering processes associated with electron-phonon interactions. Equation (A1) can subsequently be rewritten as 


$$
\begin{aligned}
G_{g}(\tau) & {\left[\hat{X}_{g}, e^{-i H_{\mathrm{sys}}^{\prime} \tau / \hbar} \hat{X}_{g} e^{i H_{\mathrm{sy}}^{\prime} \tau / \hbar} \rho(t)\right]+\text { H.c. } } \\
\simeq & \hbar^{2} g^{2}\left[G_{g}(\tau) e^{-i \Delta_{c x} t} \hat{\sigma}^{+} \hat{a} \hat{a}^{\dagger} \hat{\sigma}^{-} \rho(t)+G_{g}(\tau) e^{i \Delta_{c x} t} \hat{a}^{\dagger} \hat{\sigma}^{-} \hat{\sigma}^{+} \hat{a} \rho(t)\right]-\hbar^{2} g^{2}\left[G_{g}(\tau) e^{-i \Delta_{c x} t} \hat{a}^{\dagger} \hat{\sigma}^{-} \rho(t) \hat{\sigma}^{+} \hat{a}\right. \\
& \left.+G_{g}(\tau) e^{i \Delta_{c x} t} \hat{\sigma}^{+} \hat{a} \rho(t) \hat{a}^{\dagger} \hat{\sigma}^{-}\right]+\hbar^{2} g^{2}\left[G_{g}^{*}(\tau) e^{-i \Delta_{c x} t} \rho(t) \hat{a}^{\dagger} \hat{\sigma}^{-} \hat{\sigma}^{+} \hat{a}+G_{g}^{*}(\tau) e^{i \Delta_{c x} t} \rho(t) \hat{\sigma}^{+} \hat{a} \hat{a}^{\dagger} \hat{\sigma}^{-}\right] \\
& -\hbar^{2} g^{2}\left[G_{g}^{*}(\tau) e^{-i \Delta_{c x} t} \hat{\sigma}^{+} \hat{a} \rho(t) \hat{a}^{\dagger} \hat{\sigma}^{-}+G_{g}^{*}(\tau) e^{i \Delta_{c x} t} \hat{a}^{\dagger} \hat{\sigma}^{-} \rho(t) \hat{\sigma}^{+} \hat{a}\right],
\end{aligned}
$$

which can be further simplified to

$$
\begin{aligned}
& G_{g}(\tau)\left[\hat{X}_{g}, e^{-i H_{\mathrm{sy}}^{\prime} \tau / \hbar} \hat{X}_{g} e^{i H_{\mathrm{sys}}^{\prime} \tau / \hbar} \rho(t)\right]+\text { H.c. } \\
& \simeq \hbar^{2} g^{2} \operatorname{Re}\left[G_{g}(\tau) e^{-i \Delta_{c x} t}\right]\left(\hat{\sigma}^{+} \hat{a} \hat{a}^{\dagger} \hat{\sigma}^{-} \rho(t)+\rho(t) \hat{\sigma}^{+} \hat{a} \hat{a}^{\dagger} \hat{\sigma}^{-}-2 \hat{a}^{\dagger} \hat{\sigma}^{-} \rho(t) \hat{\sigma}^{+} \hat{a}\right)+\hbar^{2} g^{2} \operatorname{Re}\left[G_{g}(\tau) e^{i \Delta_{c x} t}\right]\left(\hat{a}^{\dagger} \hat{\sigma}^{-} \hat{\sigma}^{+} \hat{a} \rho(t)\right. \\
& \left.+\rho(t) \hat{a}^{\dagger} \hat{\sigma}^{-} \hat{\sigma}^{+} \hat{a}-2 \hat{\sigma}^{+} \hat{a} \rho(t) \hat{a}^{\dagger} \hat{\sigma}^{-}\right)+i \hbar^{2} g^{2} \operatorname{Im}\left[G_{g}(\tau) e^{-i \Delta_{c x} t}\right]\left(\hat{\sigma}^{+} \hat{a} \hat{a}^{\dagger} \hat{\sigma}^{-} \rho(t)-\rho(t) \hat{\sigma}^{+} \hat{a} \hat{a}^{\dagger} \hat{\sigma}^{-}\right) \\
& +i \hbar^{2} g^{2} \operatorname{Im}\left[G_{g}(\tau) e^{i \Delta_{c x} t}\right]\left(\hat{a}^{\dagger} \hat{\sigma}^{-} \hat{\sigma}^{+} \hat{a} \rho(t)-\rho(t) \hat{a}^{\dagger} \hat{\sigma}^{-} \hat{\sigma}^{+} \hat{a}\right) .
\end{aligned}
$$

Using $\sum_{m=g, u} G_{m}(\tau)=\langle B\rangle^{2}\left(e^{\phi(\tau)}-1\right)$, the defined scattering rates become

$$
\Gamma_{\mathrm{ph}}^{\sigma^{+} a / a^{\dagger} \sigma^{-}}=2\langle B\rangle^{2} g^{2} \operatorname{Re}\left[\int_{0}^{\infty} d \tau e^{ \pm i \Delta_{c x} \tau}\left(e^{\phi(\tau)}-1\right)\right]
$$

while the frequency shifts,

$$
\Delta_{\mathrm{ph}}^{\sigma^{+} a / a^{\dagger} \sigma^{-}}=\langle B\rangle^{2} g^{2} \operatorname{Im}\left[\int_{0}^{\infty} d \tau e^{ \pm i \Delta_{c x} \tau}\left(e^{\phi(\tau)}-1\right)\right],
$$

which are used in the Lindblad operator $L_{\mathrm{ph}}(\rho)$ and the effective Hamiltonian defined in Eq. (16).

For the case of a dot-driven system, we have an additional complication due to the coherent term driving through the exciton-phonon bath. Using similar steps as above, and also neglecting contributions involving cross terms between operators $\hat{\sigma}^{+}\left(\hat{\sigma}^{-}\right)$and $\hat{\sigma}^{+} \hat{a}\left(\hat{a}^{\dagger} \hat{\sigma}^{-}\right)$, which scale as $g \eta_{x}$, we again obtain an effective Lindblad form of the ME. We evaluate the exponential phase terms involving the full system Hamiltonian by replacing $H_{\text {sys }}^{\prime}$ with $H_{0}^{\prime}$.

[1] U. Hohenester, Optical Properties of Semiconductor Nanostructures: Decoherence Versus Quantum Control, in Handbook of Theoretical and Computational Nanotechnology edited by M. Rieth and W. Schommers (American Scientific Publishers, Valencia, 2006).

[2] N. Akopian, N. H. Lindner, E. Poem, Y. Berlatzky, J. Avron, D. Gershoni, B. D. Gerardot, and P. M. Petroff, Entangled Photon Pairs from Semiconductor Quantum Dots, Phys. Rev. Lett. 96, 130501 (2006).

[3] A. Muller, W. Fang, J. Lawall, and G. S. Solomon, Creating Polarization-Entangled Photon Pairs from a Semiconductor Quantum Dot Using the Optical Stark Effect, Phys. Rev. Lett. 103, 217402 (2009).

[4] R. B. Patel, A. J. Bennett, K. Cooper, P. Atkinson, C. A. Nicoll, D. A. Ritchie, and A. J. Shields, Postselective TwoPhoton Interference from a Continuous Nonclassical Stream of Photons Emitted by a Quantum Dot, Phys. Rev. Lett. 100, 207405 (2008).

[5] S. Ates, S. M. Ulrich, S. Reitzenstein, A. Löffler, A. Forchel, and P. Michler, Post-Selected Indistinguishable
Photons from the Resonance Fluorescence of a Single Quantum Dot in a Microcavity, Phys. Rev. Lett. 103, 167402 (2009).

[6] C. Santori, D. Fattal, J. Vučković, G. S. Solomon, and Y. Yamamoto, Indistinguishable Photons from a SinglePhoton Device, Nature (London) 419, 594 (2002).

[7] See, e.g., J.P. Reithmaier, G. Seogonk, A. Löffler, C. Hofmann, S. Kuhn, S. Reitzenstein, L. V. Keldysh, V. D. Kulakovskii, T.L. Reinecke, and A. Forchel, Strong Coupling in a Single Quantum Dot Semiconductor Microcavity System, Nature (London) 432, 197 (2004).

[8] T. Yoshie, A. Scherer, J. Hendrickson, G. Khitrova, H. M. Gibbs, G. Rupper, C. Ell, O. B. Shchekin, and D. G. Deppe, Vacuum Rabi Splitting With a Single Quantum Dot in a Photonic Crystal Nanocavity, Nature (London) 432, 200 (2004).

[9] D. Press, S. Götzinger, S. Reitzenstein, C. Hofmann, A. Löffler, M. Kamp, A. Forchel, and Y. Yamamoto, Photon Antibunching from a Single Quantum-Dot-Microcavity System in the Strong Coupling Regime, Phys. Rev. Lett. 98, 117402 (2007).

[10] A. Muller, E. B. Flagg, P. Bianucci, X. Y. Wang, D. G. Deppe, W. Ma, J. Zhang, G. J. Salamo, M. Xiao, and C. K. Shih, Resonance Fluorescence from a Coherently Driven Semiconductor Quantum Dot in a Cavity, Phys. Rev. Lett. 99, 187402 (2007).

[11] E. B. Flagg, A. Muller, J. W. Robertson, S. Founta, D. G. Deppe, M. Xiao, W. Ma, G. J. Salamo, and C. K. Shih, Resonantly Driven Coherent Oscillations in a Solid-State Quantum Emitter, Nature Phys. 5, 203 (2009).

[12] A. N. Vamivakas, Y. Zhao, C.-Y. Lu, and M. Atatüre, SpinResolved Quantum-Dot Resonance Fluorescence, Nature Phys. 5, 198 (2009).

[13] S. Ates, S. M. Ulrich, A. Ulhaq, S. Reitzenstein, A. Löffler, S. Höfling, A. Forchel, and P. Michler, NonResonant Dot-Cavity Coupling and its Potential for Resonant Single-Quantum-Dot Spectroscopy, Nat. Photon. 3, 724 (2009).

[14] A. Majumdar, E. D. Kim, Y. Gong, M. Bajcsy, and J. Vučković, Phonon Mediated Off-Resonant Quantum Dot-Cavity Coupling Under Resonant Excitation of the Quantum Dot, Phys. Rev. B 84, 085309 (2011); see also A. Majumdar, E. D. Kim, Y. Gong, M. Bajcsy, P. Petroff, and J. Vučković, Probing of Single Quantum Dot Dressed 
States via an Off-Resonant Cavity, Phys. Rev. B 84, 085310 (2011).

[15] K. Hennessy, A. Badolato, M. Winger, A. Atatüre, S. Falt, E. L. Hu, and A. Imamoglŭ, Quantum Nature of a Strongly Coupled Single Quantum Dot-Cavity System, Nature (London) 445, 896 (2007).

[16] M. Kaniber, A. Laucht, A. Neumann, J. M. Villas-Bôas, M. Bichler, M.-C. Amann, and J. J. Finley, Investigation of the Nonresonant Dot-Cavity Coupling in Two-Dimensional Photonic Crystal Nanocavities, Phys. Rev. B 77, 161303 (R) (2008).

[17] R. Oulton, B. D. Jones, S. Lam, A. R. A. Chalcraft, D. Szymanski, D. O'Brien, T. F. Krauss, D. Sanvitto, A. M. Fox, D. M. Whittaker, M. Hopkinson, and M. S. Skolnick, Polarized Quantum Dot Emission from Photonic Crystal Nanocavities studied under Mode Resonant Excitation, Opt. Express 15, 17221 (2007).

[18] J. Suffczynski, A. Dousse, K. Gauthron, A. Lemaitre, I. Sagnes, L. Lanco, J. Bloch, P. Voisin, and P. Senellart, Origin of the Optical Emission Within the Cavity Mode of Coupled Quantum Dot-Cavity Systems, Phys. Rev. Lett. 103, 027401 (2009).

[19] T. Tawara, H. Kamada, S. Hughes, H. Okamoto, M. Notomi, and T. Sogawa, Cavity Mode Emission in Weakly Coupled Quantum Dot-Cavity Systems, Opt. Express 17, 6643 (2009).

[20] Y. Ota, S. Iwamoto, N. Kumagai, and Y. Arakawa, Impact of Electron-Phonon Interactions on Quantum-Dot Cavity Quantum Electrodynamics, arXiv:0908.0788.

[21] D. Dalacu, K. Mnaymneh, V. Sazonova, P. J. Poole, G. C. Aers, J. Lapointe, R. Cheriton, A. J. SpringThorpe, and R. L. Williams, Deterministic Emitter-Cavity Coupling Using a Single-Site Controlled Quantum Dot, Phys. Rev. B 82, 033301 (2010).

[22] M. Calic, P. Gallo, M. Felici, K. A. Atlasov, B. Dwir, A. Rudra, G. Biasiol, L. Sorba, G. Tarel, V. Savona, and E. Kapon, Phonon-Mediated Coupling of InGaAs/GaAs Quantum-Dot Excitons to Photonic Crystal Cavities, Phys. Rev. Lett. 106, 227402 (2011).

[23] F. Milde, A. Knorr, and S. Hughes, Role of ElectronPhonon Scattering on the Vacuum Rabi Splitting of a Single-Quantum Dot and a Photonic Crystal Nanocavity, Phys. Rev. B 78, 035330 (2008).

[24] S. Hughes, P. Yao, F. Milde, A. Knorr, D. Dalacu, K. Mnaymneh, V. Sazonova, P. J. Poole, G. C. Aers, J. Lapointe, R. Cheriton, and R. L. Williams, Influence of Electron-Acoustic Phonon Scattering on Off-Resonant Cavity Feeding within a Strongly Coupled Quantum-Dot Cavity System, Phys. Rev. B 83, 165313 (2011).

[25] J. Xue, K-D Zhu, and H. Zheng, Detuning Effect in Quantum Dynamics of a Strongly Coupled Single Quantum Dot Cavity System, J. Phys. Condens. Matter 20, 325209 (2008).

[26] U. Hohenester, A. Laucht, M. Kaniber, N. Hauke, A. Neumann, A. Mohtashami, M. Selinger, M. Bichler, and J. J. Finley, Phonon-Assisted Transitions from Quantum Dot Excitons to Cavity Photons, Phys. Rev. B 80, 201311 (2009).

[27] U. Hohenester, Cavity Quantum Electrodynamics with Semiconductor Quantum Dots: Role of Phonon-Assisted Cavity Feeding, Phys. Rev. B 81, 155303 (2010).
[28] P. Kaer, T. R. Nielsen, P. Lodahl, A.-P. Jauho, and J. Mørk, Non-Markovian Model of Photon-Assisted Dephasing by Electron-Phonon Interactions in a Coupled Quantum-Dot-Cavity System, Phys. Rev. Lett. 104, 157401 (2010).

[29] G. Tarel and V. Savona, Linear Spectrum of a Quantum Dot Coupled to a Nanocavity, Phys. Rev. B 81, 075305 (2010).

[30] A. Majumdar, A. Faraon, E. D. Kim, D. Englund, H. Kim, P. Petroff, and J. Vučković, Linewidth Broadening of a Quantum Dot Coupled to an Off-Resonant Cavity, Phys. Rev. B 82, 045306 (2010).

[31] P. P. Paskov, P. O. Holtz, S. Wongmanerod, B. Monemar, J. M. Garcia, W. V. Schoenfeld, and P. M. Petroff, Auger Processes in InAs Self-Assembled Quantum Dots, Physica (Amsterdam) 6E, 440 (2000).

[32] M. Winger, T. Volz, G. Tarel, S. Portolan, A. Badolato, K. J. Hennessy, E. L. Hu, A. Beveratos, J. Finley, V. Savona, and A. Imamoğlu, Explanation of Photon Correlations in the Far-Off-Resonance Optical Emission from a Quantum-Dot-Cavity System, Phys. Rev. Lett. 103, 207403 (2009).

[33] R. Völkl, M. Griesbeck, S. A. Tarasenko, D. Schuh, W. Wegscheider, C. Schüller, and T. Korn, Spin Dephasing and Photoinduced Spin Diffusion in a High-Mobility TwoDimensional Electron System Embedded in a GaAs(Al,Ga)As Quantum Well Grown in the [110] Direction, Phys. Rev. B 83, 241306 (2011).

[34] A. Laucht, M. Kaniber, A. Mohtashami, N. Hauke, M. Bichler, and J.J. Finley, Temporal Monitoring of Nonresonant Feeding of Semiconductor Nanocavity Modes by Quantum Dot Multiexciton Transitions, Phys. Rev. B 81, 241302 (2010).

[35] A. Ulhaq, S. Ates, S. Weiler, S. M. Ulrich, S. Reitzenstein, A. Löffler, S. Höfling, L. Worschech, A. Forchel, and P. Michler, Linewidth Broadening and Emission Saturation of a Resonantly Excited Quantum Dot Monitored via an Off-Resonant Cavity Mode, Phys. Rev. B 82, 045307 (2010).

[36] L. Besombes, K. Kheng, L. Marsal, and H. Mariette, Acoustic Phonon Broadening Mechanism in Single Quantum Dot Emission, Phys. Rev. B 63, 155307 (2001).

[37] E. Peter, J. Hours, P. Senellart, A. Vasanelli, A. Cavanna, J. Bloch, and J. M. Gérard, Phonon Sidebands in Exciton and Biexciton Emission from Single GaAs Quantum Dots, Phys. Rev. B 69, 041307 (2004).

[38] I. Favero, G. Cassabois, R. Ferreira, D. Darson, C. Voisin, J. Tignon, C. Delalande, G. Bastard, Ph. Roussignol, and J. M. Gérard, Acoustic Phonon Sidebands in the Emission Line of Single InAs/GaAs Quantum Dots, Phys. Rev. B 68 , 233301 (2003).

[39] S. M. Ulrich, S. Ates, S. Reitzenstein, A. Löffler, A. Forchel, and P. Michler, Dephasing of Mollow Triplet Sideband Emission of a Resonantly Driven Quantum Dot in a Microcavity, Phys. Rev. Lett. 106, 247402 (2011).

[40] C. Roy and S. Hughes, Phonon-Dressed Mollow Triplet in the Regime of Cavity Quantum Electrodynamics: Excitation-Induced Dephasing and Nonperturbative Cavity Feeding Effects, Phys. Rev. Lett. 106, 247403 (2011). 
[41] D. P. S. McCutcheon and A. Nazir, Quantum Dot Rabi Rotations Beyond the Weak Exciton-Phonon Coupling Regime, New J. Phys. 12, 113042 (2010).

[42] A. Nazir, Photon Statistics from a Resonantly Driven Quantum Dot, Phys. Rev. B 78, 153309 (2008).

[43] G. D. Mahan, Many-Particle Physics (Plenum, New York, 1990).

[44] B. Krummheuer, V. M. Axt, and T. Kuhn, Theory of Pure Dephasing and the Resulting Absorption Line Shape in Semiconductor Quantum Dots, Phys. Rev. B 65, 195313 (2002).

[45] I. Wilson-Rae and A. Imamoğlu, Quantum Dot CavityQED in the Presence of Strong Electron-Phonon Interactions, Phys. Rev. B 65, 235311 (2002).

[46] A. Würger, Strong-Coupling Theory for the Spin-Phonon Model, Phys. Rev. B 57, 347 (1998).

[47] We remark that, in fact, for our cw studies, the more complicated nonlocal ME yields the same result as our simpler time-local ME. We will show this directly in a future publication.

[48] D. Mogilevtsev, A. P. Nisovtsev, S. Kilin, S. B. Cavalcanti, H.S. Brandi, and L.E. Oliveira, Driving-Dependent Damping of Rabi Oscillations in Two-Level Semiconductor Systems, Phys. Rev. Lett. 100, 017401 (2008).

[49] A. J. Ramsay, A. V. Gopal, E. M. Gauger, A. Nazir, B. W. Lovett, A.M. Fox, and M.S. Skolnick, Damping of Exciton Rabi Rotations by Acoustic Phonons in Optically Excited InGaAs/GaAs Quantum Dots, Phys. Rev. Lett. 104, 017402 (2010).

[50] H. J. Carmichael and D.F. Walls, Master Equation for Strongly Interacting Systems, J. Phys. A 6, 1552 (1973).

[51] M. Florescu and S. John, Single-Atom Switching in Photonic Crystals, Phys. Rev. A 64, 033801 (2001).

[52] A. Kowalewska-Kudlaszyk and R. Tanas, Generalized Master Equation for a Two-Level atom in a Strong Field and Tailored Reservoirs, J. Mod. Opt. 48, 347 (2001).

[53] P. Borri, W. Langbein, S. Schneider, U. Woggon, R. L. Sellin, D. Ouyang, and D. Bimberg, Ultralong Dephasing Time in InGaAs Quantum Dots, Phys. Rev. Lett. 87, 157401 (2001).

[54] S. Rudin, T. L. Reinecke, and M. Bayer, Temperature Dependence of Optical Linewidth in Single InAs Quantum Dots, Phys. Rev. B 74, 161305(R). (2006).

[55] E. A. Muljarov and R. Zimmermann, Dephasing in Quantum Dots: Quadratic Coupling to Acoustic Phonons, Phys. Rev. Lett. 93, 237401 (2004).

[56] M. Bayer and A. Forchel, Temperature Dependence of the Exciton Homogeneous Linewidth in $\mathrm{In}_{0.60} \mathrm{Ga}_{0.40} \mathrm{As} / \mathrm{GaAs}$ Self-Assembled Quantum Dots, Phys. Rev. B 65, 041308 (2002).
[57] P. Machnikowski, Change of Decoherence Scenario and Appearance of Localization due to Reservoir Anharmonicity, Phys. Rev. Lett. 96, 140405 (2006).

[58] E. A. Muljarov and R. Zimmermann, Dephasing in Quantum Dots: Quadratic Coupling to Acoustic Phonons, Phys. Rev. Lett. 93, 237401 (2004).

[59] G. Ortner, D. R. Yakovlev, M. Bayer, S. Rudin, T. L. Reinecke, S. Fafard, Z. Wasilewski, and A. Forchel, Temperature Dependence of the Zero-Phonon Linewidth in InAsGaAs Quantum Dots, Phys. Rev. B 70, 201301(R) (2004).

[60] S. Rudin, T. L. Reinecke, and M. Bayer, Temperature Dependence of Optical Linewidth in Single InAs Quantum Dots, Phys. Rev. B 74, 161305(R) (2006).

[61] G. Lindwall, A. Wacker, C. Weber, and A. Knorr, ZeroPhonon Linewidth and Phonon Satellites in the Optical Absorption of Nanowire-Based Quantum Dots, Phys. Rev. Lett. 99, 087401 (2007).

[62] C. Roy and S. John, Microscopic Theory of MultiplePhonon-Mediated Dephasing and Relaxation of Quantum Dots Near a Photonic Band Gap, Phys. Rev. A 81, 023817 (2010).

[63] H.-P. Breuer, B. Kappler, and F. Petruccione, Stochastic Wave-Function Method for Non-Markovian Quantum Master Equations, Phys. Rev. A 59, 1633 (1999).

[64] We use the following phonon parameters for our calculations: deformation potentials $D_{e}-D_{h}=6.5 \mathrm{eV}$, mass density $\rho=5.667 \mathrm{~g} \mathrm{~cm}^{-3}$, longitudinal sound velocity $c_{l}=3800 \mathrm{~m} \mathrm{~s}^{-1}$. We find $\omega_{b}=1 \mathrm{meV}$ and $\alpha_{p} /(2 \pi)^{2}=0.06 \mathrm{ps}^{2}$ as typical numbers for InAs/GaAs quantum dots [25], which we have also used to fit several semiconductor cavity-QED experiments [24,40].

[65] D. P. S. McCutcheon, N. S. Dattani, E. M. Gauger, B. W. Lovett, and A. Nazir, A General Approach to Quantum Dynamics Using a Variational Master Equation: Application to Phonon-Damped Rabi Rotations in Quantum Dots, Phys. Rev. B 84, 081305(R) (2011).

[66] N. Makri and D.E. Makarov, Tensor Propagator for Iterative Quantum Time Evolution of Reduced Density Matrices. I. Theory, J. Chem. Phys. 102, 4600 (1995).

[67] N. Makri and D.E. Makarov, Tensor Propagator for Iterative Quantum Time Evolution of Reduced Density Matrices. II. Numerical Methodology, J. Chem. Phys. 102, 4611 (1995).

[68] S. M. Tan, A Computational Toolbox for Quantum and Atomic Optics, J. Opt. B 1, 424 (1999).

[69] K. J. Ahn, J. Förstner, and A. Knorr, Resonance Fluorescence of Semiconductor Quantum Dots: Signatures of the Electron-Phonon Interaction, Phys. Rev. B 71, 153309 (2005). 